STRUCTURAL BIOLOGY

ISSN 2059-7983

Received 13 November 2021

Accepted 27 January 2022

Edited by G. Cingolani, Thomas Jefferson University, USA

₹ These authors contributed equally. $\S$ Present address: Laboratory of Applied Biotechnology, Department of Biotechnology, Ghent University, Ghent, Belgium.

Keywords: endolysins; phage lysins; Pae87; bacteriophages; antimicrobial peptides; protein structure; Pseudomonas aeruginosa; carbohydrate-active enzymes.

PDB references: Pseudomonas aeruginosa bacteriophage JG004 endolysin Pae87, apo form, $7 \mathrm{q} 4 \mathrm{~s}$; ligand-bound, $7 \mathrm{q} 4 \mathrm{t}$

Supporting information: this article has supporting information at journals.iucr.org/d
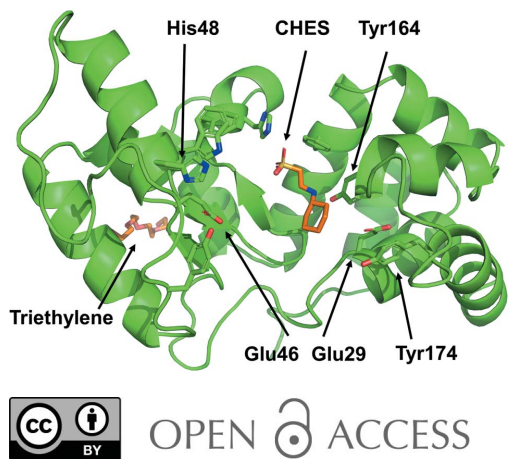

\section{Monomodular Pseudomonas aeruginosa phage JG004 lysozyme (Pae87) contains a bacterial surface-active antimicrobial peptide-like region and a possible substrate-binding subdomain}

\author{
Roberto Vázquez, ${ }^{\mathrm{a}, \mathrm{b}} \neq \S$ Mateo Seoane-Blanco, ${ }^{\mathrm{c}} \neq$ Virginia Rivero-Buceta, ${ }^{\mathrm{a}, \mathrm{d}}$ \\ Susana Ruiz, ${ }^{a, b}$ Mark J. van Raaij ${ }^{\mathrm{c}}$ and Pedro García ${ }^{\mathrm{a}, \mathrm{b}}$ *
}

${ }^{\mathbf{a}}$ Microbial and Plant Biotechnology Department, Centro de Investigaciones Biológicas Margarita Salas (CIB-CSIC), Madrid, Spain, bentro de Investigación Biomédica en Red de Enfermedades Respiratorias (CIBERES), Madrid, Spain, ${ }^{\mathbf{c}}$ Department of Macromolecular Structure, Centro Nacional de Biotecnología (CNB-CSIC), Madrid, Spain, and

dinterdisciplinary Platform for Sustainable Plastics, Madrid, Spain. *Correspondence e-mail: pgarcia@cib.csic.es

Phage lysins are a source of novel antimicrobials to tackle the bacterial antibiotic-resistance crisis. The engineering of phage lysins is being explored as a game-changing technological strategy to introduce a more precise approach in the way in which antimicrobial therapy is applied. Such engineering efforts will benefit from a better understanding of lysin structure and function. In this work, the antimicrobial activity of the endolysin from Pseudomonas aeruginosa phage JG004, termed Pae87, has been characterized. This lysin had previously been identified as an antimicrobial agent candidate that is able to interact with the Gram-negative surface and disrupt it. Further evidence is provided here based on a structural and biochemical study. A high-resolution crystal structure of Pae87 complexed with a peptidoglycan fragment showed a separate substratebinding region within the catalytic domain, $18 \AA$ away from the catalytic site and located on the opposite side of the lysin molecule. This substrate-binding region was conserved among phylogenetically related lysins lacking an additional cellwall-binding domain, but not among those containing such a module. Two glutamic acids were identified to be relevant for the peptidoglycan-degradation activity, although the antimicrobial activity of Pae87 was seemingly unrelated. In contrast, an antimicrobial peptide-like region within the Pae87 C-terminus, named P87, was found to be able to actively disturb the outer membrane and display antibacterial activity by itself. Therefore, an antimicrobial mechanism for Pae87 is proposed in which the P87 peptide plays the role of binding to the outer membrane and disrupting the cell-wall function, either with or without the participation of the catalytic activity of Pae87.

\section{Introduction}

Antibiotic resistance is becoming one of the most serious threats to public health worldwide, as the number of multiresistant bacterial strains is growing progressively. Therefore, the search for alternative treatments to standard antibiotics to fight these pathogenic 'superbugs' is becoming more urgent (O'Neill, 2016). Bacteriophage (phage) lysins are a promising strategy that is currently viewed as a feasible approach towards the development of novel, potentially marketable antibacterial agents (Abdelkader et al., 2019). Lysins are highly evolved enzymes produced by phages at the end of the lytic infection cycle to degrade the bacterial peptidoglycan, leading to cell lysis and phage progeny release. For about twenty years, phage lysins have been widely investigated as 
novel antimicrobial agents to treat bacterial infections mainly caused by Gram-positive pathogens. This mechanism relies on adding the purified enzyme exogenously ('lysis from without') and provokes the rapid degradation of the substrate (the peptidoglycan) and thus the lysis and death of susceptible bacteria, including multi-drug-resistant strains. Lysins have demonstrated several advantages over standard antibiotics, including (i) rapid killing activity against both stationary- and exponential-phase bacteria, practically within a few minutes of contact with the peptidoglycan substrate, (ii) effectiveness against multi-drug-resistant bacteria, (iii) specificity to the target pathogen, especially against Gram-positive bacteria, which allows the preservation of the normal microbiota, (iv) a seemingly very unlikely appearance of resistance, probably due to the conservation of its substrate, the peptidoglycan, (v) synergistic effects with other lysins or antibiotics and (vi) efficient lethal activity against colonizing pathogens growing on mucosal surfaces and/or in biofilms (Pastagia et al., 2013).

The architecture of lysins varies depending on their origin, but those from phages infecting Gram-positive bacteria usually have a modular structure consisting of one or two catalytic domains, which harbour lytic activity against the host species, and a C-terminal cell-wall-binding domain (CWBD), which recognizes a cell-wall traits specific to the bacteria that it targets. On the contrary, the great majority of lysins from phages infecting Gram-negative bacteria display a globular organization containing only one catalytic domain (Vázquez, García et al., 2021). The catalytic domains are responsible for the cleavage of a specific bond within the peptidoglycan, and based on the bond that they break lysins can be classified as glycosidases, $N$-acetylglucosamine amidases (NAM-amidases) or endopeptidases (Dams \& Briers, 2019). Among glycosidase lysins, which are those that act within the glycan strand of $\mathrm{N}$-acetylglucosamine (NAG) and $\mathrm{N}$-acetylmuramic acid (MurNAc), two subclasses can be recognized. $N$-Acetylmuramidases, which are termed muramidases or lysozymes, hydrolyze the bond between MurNAc and NAG at the reducing side of the former, while $N$-acetylglucosaminidases break the bond between NAG and MurNAc at the reducing side of NAG. The CWBDs that many lysins bear are responsible for the specific recognition of the insoluble substrate and the high-affinity binding of these enzymes to susceptible bacteria (Guillén et al., 2010; Low et al., 2011).

In a previous study (Vázquez, Blanco-Gañán et al., 2021), the lysin from Pseudomonas aeruginosa phage JG004, termed Pae87, was mined from a data set of phage lysin sequences (Vázquez, García et al., 2021; Vázquez et al., 2020) on the basis of containing a putative C-terminal positively charged, antimicrobial peptide (AMP)-like region. The presence of such a region was probed under the hypothesis that it would enable Pae87 to disrupt the Gram-negative outer membrane (OM), which is often cited as a barrier to lysin activity from without (Briers \& Lavigne, 2015). Pae87 was demonstrated to have an antimicrobial effect against $P$. aeruginosa and some other Gram-negative pathogens (Vázquez, Blanco-Gañán et al., 2021). Nowadays, insightful knowledge into the structure of lysins, as well as implications for their function (either as exogenous antimicrobials or as lysis effectors for the release of virion particles from the host cell), is a must in order to engage in protein-based antimicrobial engineering. An example is the combinatorial engineering of lysin modules or the derivation of AMPs from lysins (Duyvejonck et al., 2021; Thandar et al., 2016). Therefore, in this work we provide a fine characterization of the structural elements that contribute to the observed activities of Pae87. We do so by (i) obtaining the crystal structure of the protein, (ii) examining putative catalytic residues by point mutation and (iii) testing the antimicrobial and membrane-permeabilizing activity of Pae87 and the AMP derived from its C-terminal region, named P87.

\section{Materials and methods}

\subsection{Bacterial strains, media and growth conditions}

All of the Gram-negative bacteria used in this work (P. aeruginosa, Escherichia coli, Acinetobacter baumannii, Acinetobacter pitti and Klebsiella pneumoniae) were grown in lysogeny broth (LB, NZYTech) at $37^{\circ} \mathrm{C}$ with aeration (shaking at $200 \mathrm{rev} \mathrm{min}^{-1}$ ), except for Moraxella catarrhalis. This bacterial species, plus some Gram-positive species (Staphylococcus aureus, Streptococcus pyogenes and Streptococcus Milleri group strain), were cultured in brain heart infusion broth (BHI, Condalab) at $37^{\circ} \mathrm{C}$. M. catarrhalis and S. aureus were shaken at $200 \mathrm{rev} \mathrm{min}^{-1}$ when grown in liquid culture. Streptococcus pneumoniae was grown in $\mathrm{C}$ medium adjusted to $\mathrm{pH} 8.0$ (Lacks \& Hotchkiss, 1960) supplemented with $0.08 \%$ yeast extract $(\mathrm{C}+\mathrm{Y})$ at $37^{\circ} \mathrm{C}$ without shaking. For solid cultures, all Gram-positive species and M. catarrhalis were grown on blood agar plates, while Gram-negative species were grown on LB agar. Details of the bacterial strains used in this work can be found in Vázquez, Blanco-Gañán et al. (2021).

\subsection{Plasmids, oligonucleotides and overlap extension mutagenesis}

The plasmids and oligonucleotides used throughout this work are given in Supplementary Table S1. As explained in Vázquez, Blanco-Gañán et al. (2021), a synthetic gene encoding Pae87 (pae87) was obtained from GenScript and was cloned into a pET-28a(+) vector in frame with an N-terminal $6 \times$ His tag. This expression plasmid, named pET-PA87, was heat-shock-transformed into $E$. coli BL21(DE3) cells. For the construction of pae87 mutants (E29A, E46A and E29A/ E46A), an overlap extension PCR protocol was performed. Briefly, the pET-PA87 plasmid was used as a template for two separate PCR reactions per mutation. Each of these reactions amplified a fragment of the pae 87 gene in such a way that both fragments shared an overlapping section $(\sim 20 \mathrm{nt})$ in which the mutated bases were located. A third PCR reaction was then performed using the pae87 flanking primers pae87_f and pae87_ $3^{\prime}$ and a mixture of the resulting amplification products from the previous step as a template. The final PCR products were purified, digested with NdeI and HindIII, cloned into a pre-digested $\mathrm{pET}-28 \mathrm{a}(+)$ vector and transformed into $E$. coli 
DH10B cells. Colonies were screened by PCR and vectors putatively bearing the mutated gene were sequenced (Secugen, Centro de Investigaciones Biológicas Margarita Salas, Madrid, Spain). The final vectors were transformed into E. coli BL21(DE3) cells for expression.

\subsection{Protein expression and purification}

For the production of recombinant Pae87-based proteins, the appropriate strains were cultured in $11 \mathrm{LB}$ in the presence of $50 \mu \mathrm{g} \mathrm{ml}^{-1}$ kanamycin to an $\mathrm{OD}_{600}$ of $\sim 0.6-0.8$. Expression was then induced with $0.4 \mathrm{~m} M$ isopropyl $\beta$-D-1-thiogalactopyranoside and incubation was resumed at $20^{\circ} \mathrm{C}$ for up to $48 \mathrm{~h}$. After centrifugation $\left(12000 \mathrm{~g}, 20 \mathrm{~min}, 4^{\circ} \mathrm{C}\right)$, the pelleted biomass was resuspended in $\sim 30 \mathrm{ml} 20 \mathrm{~m} M$ sodium phosphate buffer (NaPiB) pH 7.4 containing $0.3 M \mathrm{NaCl}$ and $40 \mathrm{~m} M$ imidazole. These cell suspensions were disrupted by sonication and the cell debris was separated again by centrifugation $\left(18000 \mathrm{~g}, 20 \mathrm{~min}, 4^{\circ} \mathrm{C}\right)$. The supernatants containing the protein extracts were then applied onto a HisTrap FF $5 \mathrm{ml}$ column (GE Healthcare) loaded with nickel ions using an ÄKTA start liquid-chromatography machine (GE Healthcare). After a thorough washing step using the resuspension buffer, $20 \mathrm{~m} M \mathrm{NaPiB}$ pH 7.4 containing $0.3 \mathrm{M} \mathrm{NaCl}$ and $0.5 M$ imidazole was used to elute the purified protein. The buffer of the purified fractions was exchanged to $20 \mathrm{~m} M \mathrm{NaPiB} \mathrm{pH} 7.4$ containing $150 \mathrm{mM} \mathrm{NaCl}$ prior to assaying the proteins using HiTrap Desalting $5 \mathrm{ml}$ columns. The concentration of the purified proteins was estimated from $A_{280}$ measurements using predicted molar extinction coefficients (Supplementary Table S2). Protein samples were maintained at $4^{\circ} \mathrm{C}$ for up to a month without apparent signs of precipitation or loss of activity.

\subsection{Synthesis and quantification of peptide P87}

Peptide P87 (LNTFVRFIKINPAIHKALKSKNWAEFAKR) was synthesized and provided by GenScript as a freeze-dried powder. It was dissolved in distilled water and the concentration was estimated by measuring the $A_{280}$ using a molar extinction coefficient of $5500 M^{-1} \mathrm{~cm}^{-1}$ as predicted by ProtParam (Wilkins et al., 1999). Diluted peptide aliquots were kept at $-20^{\circ} \mathrm{C}$.

\subsection{P. aeruginosa PAO1 peptidoglycan purification and muralytic activity assay}

$P$. aeruginosa PAO1 peptidoglycan purification and a dyerelease muralytic activity assay were conducted essentially as described in Vázquez, Blanco-Gañán et al. (2021). Briefly, $P$. aeruginosa PAO1 cells were cultured in $11 \mathrm{LB}$ broth until the $\mathrm{OD}_{600}$ reached 0.8-1.0. The culture was then centrifuged $\left(4000 \mathrm{~g}, 15 \mathrm{~min}, 4^{\circ} \mathrm{C}\right)$ and resuspended in $20 \mathrm{ml} \mathrm{PBS} .80 \mathrm{ml} 5 \%$ SDS was added and the mixture was boiled for $30 \mathrm{~min}$ with vigorous shaking. After overnight incubation at room temperature, the suspension was ultracentrifuged $(100000 \mathrm{~g}$ for $60 \mathrm{~min}$ at $20^{\circ} \mathrm{C}$ ) and the pellet was resuspended in distilled water and subjected to dialysis against water for 24-72 h to wash out as much SDS as possible. The samples were then ultracentrifuged in the same conditions as before and washed again as many times as necessary to remove all SDS (typically 1-3 more times, until a thick foam layer was no longer formed upon resuspension). RNA, DNA and proteins were then eliminated by successive treatments with RNAse, DNAse and trypsin as described in Vázquez, Blanco-Gañán et al. (2021). Finally, the peptidoglycan sacculi were ultracentrifuged again, the supernatant was removed and the pellet was dried for 24$48 \mathrm{~h}$ at $37^{\circ} \mathrm{C}$ to determine the dry weight yield of the process (typically $12 \mathrm{mg}$ per litre of initial culture).

Purified sacculi were dyed by resuspending them in freshly prepared 0.02 $M$ Remazol Brilliant Blue (RBB) solution in $0.2 M$ sodium hydroxide. An incubation of about $6 \mathrm{~h}$ was conducted at $37^{\circ} \mathrm{C}$ with shaking followed by overnight incubation at $4{ }^{\circ} \mathrm{C}$. After staining, several ultracentrifugation and washing steps with distilled water were conducted until the supernatants were clear (usually 3-4 washing steps). The resuspension water volume of the final pellets was adjusted to an $A_{595}$ of $\sim 1.5$. For the dye-release assay, $100 \mu \mathrm{l}$ of the RBBstained sacculi were centrifuged $\left(12000 \mathrm{~g}, 20 \mathrm{~min}, 20^{\circ} \mathrm{C}\right)$ and the supernatant was discarded. The pelleted sacculi were then resuspended in $100 \mu \mathrm{l}$ of a solution of $\mathrm{NaPiB} \mathrm{pH} 6.0$ containing $150 \mathrm{mM} \mathrm{NaCl}$ and the desired concentration of enzyme or just buffer for the control. The samples were incubated for $10 \mathrm{~min}$ at $37^{\circ} \mathrm{C}$ and reactions were stopped by incubation for a further $5 \mathrm{~min}$ at $95^{\circ} \mathrm{C}$. The samples were then centrifuged $\left(12000 \mathrm{~g}, 20 \mathrm{~min}, 20^{\circ} \mathrm{C}\right)$ and the $A_{595}$ of the supernatants was determined using a VersaMax multi-well plate spectrophotometer (Molecular Devices).

\subsection{Antimicrobial activity assays}

Antimicrobial activity assays were performed by incubating a resting bacterial cell suspension in $\mathrm{NaPiB}$ pH 6.0 with $150 \mathrm{mM} \mathrm{NaCl}$ at $37^{\circ} \mathrm{C}$ together with the corresponding antibacterial protein or peptide (Vázquez, Blanco-Gañán et al., 2021). Bacteria at the mid-to-late exponential phase, as evaluated by turbidimetry, were harvested by centrifugation $\left(3000 \mathrm{~g}, 10 \mathrm{~min}, 4^{\circ} \mathrm{C}\right)$. The pelleted cells were resuspended in half the volume of buffer and pipetted onto a 96-well plate (100 $\mu \mathrm{l}$ per well). $100 \mu \mathrm{l}$ of the same buffer containing the desired concentration of the compound to be tested was then added and the plate was incubated at $37^{\circ} \mathrm{C}$ for $2 \mathrm{~h}$. Several measurements were performed on the treated resting cells, namely $\mathrm{OD}_{600}$ monitoring, viable cell counts by plating tenfold serial dilutions at the end of the experiment and observation under a fluorescence microscope [Leica DM4000B with an HC PL APO $100 \times / 1.40$ oil objective and L5 (bandpass 480/40) and N2.1 (515/60) filters] stained with the BacLight LIVE/DEAD bacterial viability kit L-13152 (Invitrogen-Molecular Probes, containing SYTO9 and propidium iodide).

\subsection{Analysis of degradation products}

To analyze the degradation products that resulted from Pae87 activity, a similar protocol to that described in Alvarez et al. (2016) was followed. $100 \mu \mathrm{l}$ of the P. aeruginosa PAO1 purified peptidoglycan was centrifuged $(12000 \mathrm{~g}, 20 \mathrm{~min}$, room temperature) and resuspended again in the same volume of a 
suitable reaction buffer $(20 \mathrm{~m} M \mathrm{NaPiB}$ pH $6.5,100 \mathrm{mM} \mathrm{NaCl}$ for Pae87 or $50 \mathrm{mM} \mathrm{NaPiB} \mathrm{pH} 4.9$ for the positive control cellosyl). $10 \mu \mathrm{g}$ of the corresponding enzyme (or an equivalent volume of water for the negative control) was then added and the samples were incubated overnight at $37^{\circ} \mathrm{C}$. Reactions were stopped by incubation at $98^{\circ} \mathrm{C}$ for $5 \mathrm{~min}$. The tubes were then centrifuged $(12000 \mathrm{~g}, 20 \mathrm{~min}$, room temperature) and the supernatants were collected. $0.5 \mathrm{M}$ borate buffer $\mathrm{pH} 9.0$ was added to adjust the $\mathrm{pH}$ of the samples to $8.5-9.0$ and $10 \mu \mathrm{l}$ of freshly prepared $2 \mathrm{M} \mathrm{NaBH}_{4}$ were added to reduce the sample at room temperature for $30 \mathrm{~min}$. Next, the $\mathrm{pH}$ was adjusted to $2.0-4.0$ with $25 \%$ orthophosphoric acid. The soluble muropeptides of each sample were then separated by reverse-phase high-performance liquid chromatography (RP-HPLC) on a Kinetex C18 Column $(1.7 \mu \mathrm{m}, 100 \AA ̊ .150 \times 2.1 \mathrm{~mm}$, Phenomenex) coupled to an LXQ mass spectrometer equipped with a linear ion trap (Finnigan LXQ, Thermo Scientific). Ionization was achieved by electrospray. Muropeptides were eluted at a flow rate of $0.4 \mathrm{ml} \mathrm{min}^{-1}$ with the following elution gradient: $t=0 \mathrm{~min}, 95 \% A ; t=0.5 \mathrm{~min}, 93 \%$ $A ; t=3 \mathrm{~min}, 82 \% A ; t=11 \mathrm{~min}, 50 \% A ; t=12 \mathrm{~min}, 50 \% A$; $t=12.1 \mathrm{~min}, 95 \% A ; t=15 \mathrm{~min}, 95 \% A ; A$ is $0.1 \%$ formic acid in water and $B$ is $0.1 \%$ formic acid in $40 \%$ acetonitrile. Mass spectrometry (MS) was performed using a double-play mode in which the instrument was set to acquire a full MS scan $(\mathrm{m} / \mathrm{z}$ $=150-2000)$ and a collision-induced dissociation (CID) spectrum on selected ions from the full MS scan. Spectra were analyzed using the Xcalibur software (Thermo Scientific).

\subsection{Pae87 production and crystallization}

E. coli BL21(DE3) cells transformed with pET-PA87 were grown at $37^{\circ} \mathrm{C}$ as described in Section 2.3. After reaching an $\mathrm{OD}_{600}$ of about 0.6 , cultures were cooled and Pae87 expression was induced with $0.5 \mathrm{~m} M$ isopropyl $\beta$-D-1-thiogalactopyranoside at $20^{\circ} \mathrm{C}$ overnight. The cells were centrifuged at $6000 \mathrm{~g}$ for $10 \mathrm{~min}$ at $4^{\circ} \mathrm{C}$ and the pellets were stored frozen. Later, the pellets from 11 culture were resuspended in $10 \mathrm{ml}$ lysis buffer (20 mM Tris pH 7.5, $0.5 M \mathrm{NaCl}, 20 \mathrm{~m} M$ imidazole, $5 \%$ glycerol). The cells were then disrupted with a Digital Sonifier 250 (Branson) and centrifuged at $15000 \mathrm{~g}$ for $45 \mathrm{~min}$ at $4^{\circ} \mathrm{C}$. Supernatants from centrifugation of the disrupted cells were incubated with $2 \mathrm{ml}$ nickel-nitrilotriacetic acid (Ni-NTA) agarose (Jena Bioscience) on ice for $30 \mathrm{~min}$. The mixture was then poured into an Econo-Pac Chromatography Column (Bio-Rad) and eluted by gravity. After a two-column-volume wash with lysis buffer, the protein was eluted by passing $2 \mathrm{ml}$ elution buffer $(20 \mathrm{~m} M$ Tris $\mathrm{pH} 7.5,0.5 \mathrm{M} \mathrm{NaCl}, 0.5 \mathrm{~m} M$ imidazole, $5 \%$ glycerol) six times. After analysis by denaturing gel electrophoresis, the protein-containing fractions were dialyzed in a cellulose membrane tube $(14 \mathrm{kDa}$ molecularweight cutoff, Merck-Millipore), first against $1120 \mathrm{mM}$ Tris$\mathrm{HCl} \mathrm{pH} \mathrm{7.5,} 0.2 \mathrm{M} \mathrm{NaCl}$ for 3-4 $\mathrm{h}$ at $4^{\circ} \mathrm{C}$ and then against 11 $20 \mathrm{~m} M$ Tris- $\mathrm{HCl} \mathrm{pH} 7.5$ overnight at $4^{\circ} \mathrm{C}$. Prior to ionexchange chromatography, the dialyzed sample was centrifuged at $15000 \mathrm{~g}$ for $10 \mathrm{~min}$ at $4^{\circ} \mathrm{C}$ to remove aggregates. Chromatography was performed using an ÄKTApurifier 10
FPLC system (Cytiva) with a RESOURCE Q $6 \mathrm{ml}$ column. Proteins were eluted in a gradient of $0-1 M \mathrm{NaCl}$ buffer with $20 \mathrm{~m} M$ Tris- $\mathrm{HCl} \mathrm{pH}$ 7.5. Pae87 eluted at approximately $0.18 \mathrm{M} \mathrm{NaCl}$. Fractions containing pure Pae87 were desalted and concentrated using Amicon Ultra centrifugal filters with 3 or $10 \mathrm{kDa}$ molecular-weight cutoff (Millipore). Concentrated samples were centrifuged at $15000 \mathrm{~g}$ for $10 \mathrm{~min}$ prior to crystallization trials; the pellet was discarded. The Pae87 concentration was estimated by measuring the $A_{280}$ using the predicted molar extinction coefficient (Supplementary Table S2).

Proteins were crystallized using the sitting-drop vapourdiffusion technique in MCR crystallization plates (SWISSCI). Reservoirs were filled with $50 \mu \mathrm{l}$ of various crystallization solutions. Drops consisted of $1 \mu \mathrm{l}$ protein solution (in $20 \mathrm{mM}$ Tris- $\mathrm{HCl} \mathrm{pH}$ 7.5) plus $0.5 \mu \mathrm{l}$ crystallization solution. The best apoprotein crystal was obtained at $11 \mathrm{mg} \mathrm{ml}^{-1}$ Pae87 when $20 \%(w / v)$ polyethylene glycol 8000 and $0.1 M$ CHES-NaOH $\mathrm{pH} 9.5$ was used as the crystallization solution. For the ligandprotein complex crystallization assay, $3 \mathrm{mg}$ of the dried $P$. aeruginosa $\mathrm{PAO} 1$ peptidoglycan were solubilized by adding $120 \mu \mathrm{l}$ of the purified Pae7 protein at $11 \mathrm{mg} \mathrm{ml}^{-1}$ in $20 \mathrm{mM}$ Tris- $\mathrm{HCl} \mathrm{pH} \mathrm{7.5.} \mathrm{The} \mathrm{mixture} \mathrm{was} \mathrm{centrifuged} \mathrm{at} 15000 \mathrm{~g}$ for $10 \mathrm{~min}$ at $4^{\circ} \mathrm{C}$ to remove aggregates. The supernatant was then used in crystallization trials. The best crystal appeared when $0.1 \mathrm{~m} M$ Tris- $\mathrm{HCl}$ pH $8.0,20 \%(w / v)$ polyethylene glycol monomethyl ether $5000,8 \%(v / v)$ ethylene glycol was used as the crystallization solution.

\subsection{Crystallographic data collection, structure determination and analysis}

Diffraction data were obtained on the XALOC beamline at the ALBA-CELLS synchrotron, Cerdanyola del Vallès, Spain (Juanhuix et al., 2014) using a Dectris PILATUS 6M detector. Diffraction images were processed using the CCP4 suite (Winn et al., 2011). xia2/DIALS (Winter, 2010) was used to index and integrate the images, POINTLESS (Evans, 2006) was used for space-group determination and AIMLESS (Evans \& Murshudov, 2013) was used for scaling. The crystal structure of the apo form was determined by molecular replacement with MOLREP (Vagin \& Teplyakov, 2010) using PDB entry $5 \mathrm{~nm} 7$ as the search model (Maciejewska et al., 2017). The protein model was modified using the graphics program Coot (Emsley et al., 2010) and was refined with REFMAC5 (Murshudov et al., 2011). The protein-ligand complex structure was similarly obtained by molecular replacement using molecule $A$ of the apo-form model as the search model. The resolution limit for the data to be included was determined by paired refinement with $P D B_{-} R E D O$ (Joosten et al., 2014). Models were validated with MolProbity (http://molprobity.biochem.duke.edu/; Williams et al., 2018). PyMOL (version 1.8; Schrödinger) was used for analysis and visualization of the models. Structure comparison was performed using the DALI server (Holm, 2020) and oligomerization parameters (accessible and buried surfaces, estimated dissociation energies) were analyzed with QtPISA (Krissinel, 2015). 


\subsection{Bioinformatic analyses}

The lysin sequence data set published in Vázquez, García et al. (2021) and available from Vázquez et al. (2020) was used as a sample for various analyses. Multiple sequence alignments were performed using Clustal Omega as implemented at the EMBL-EBI (Madeira et al., 2019) and were visualized using JalView (Waterhouse et al., 2009). HeliQuest (Gautier et al., 2008) and EMBOSS: charge (Rice et al., 2000) were used to predict the physicochemical properties of protein helices and the local charge plots of amino-acid sequences. To specifically determine the sequence of the AMP-like fragment at the C-terminus of Pae87, a score variable was calculated consisting of the half-sum of the min-max standardized net charge (NC) and hydrophobic moment (HM) for each 11amino acid fragment of Pae87,

$$
\frac{1}{2}\left[\frac{\mathrm{HM}_{i}-\min (\mathrm{HM})}{\max (\mathrm{HM})-\min (\mathrm{HM})}\right]+\frac{1}{2}\left[\frac{\mathrm{NC}_{i}-\min (\mathrm{NC})}{\max (\mathrm{NC})-\min (\mathrm{NC})}\right],
$$

where $\mathrm{HM}_{i}$ and $\mathrm{NC}_{i}$ are specific values of $\mathrm{HM}$ or $\mathrm{NC}$, respectively, and $\mathrm{HM}$ and $\mathrm{NC}$ are the respective sets of values for each variable. In this way, the score measured the magnitude of both parameters with equal weight and on a $0-1$ scale.

Sequence-similarity networks (SSNs) were generated to visually assess the similarity clustering of sequence sets. For this purpose, the Enzyme Similarity Tool from the Enzyme Function Initiative server (EFI-EST) was employed (Zallot et al., 2019). Briefly, this tool performs a local alignment, from which every possible pair of sequences receives a score similar to the $E$-value obtained from a typical $B L A S T$ analysis. A threshold score value was selected for the SSN so that below such a threshold sequence pairs were considered to be nonsimilar and therefore the pair would not be connected in the resulting representation. The score was selected so that sequence pairs with a similarity below $30-40 \%$ were deemed to be nonsimilar. The SSN graphs were produced using Cytoscape 3 with identity percentage-based Prefuse Force Directed layout (Shannon et al., 2003).

\subsection{Circular dichroism}

Circular-dichroism spectra were acquired at $4^{\circ} \mathrm{C}$ in $20 \mathrm{mM}$ $\mathrm{NaPiB} \mathrm{pH}$ 6.5, $150 \mathrm{~m} M \mathrm{NaCl}$ using a Jasco J700 spectropolarimeter equipped with a temperature-controlled holder. Far-UV spectra were recorded from 260 to $200 \mathrm{~nm}$ in a $1 \mathrm{~mm}$ path-length quartz cuvette. Each spectrum was obtained by averaging five accumulations collected at a scan rate of $50 \mathrm{~nm} \mathrm{~min}{ }^{-1}$ with $2 \mathrm{~s}$ response time. Buffer spectra were subtracted from $0.1 \mathrm{mg} \mathrm{ml}^{-1}$ peptide spectra and the molar ellipticity per residue was calculated. Far-UV spectra collected with different concentrations of 2,2,2-trifluoroethanol (TFE) were used to predict the ability of peptides to form secondary structures in the presence of biological membranes.

\subsection{Outer membrane $(\mathrm{OM})$ permeabilization assay}

The physiological effects of the interaction of proteins or peptides with the $P$. aeruginosa PAO1 OM were assayed using $N$-phenyl-1-naphthylamine (NPN) as a fluorescent probe (Loh et al., 1984; Helander \& Mattila-Sandholm, 2000). The assay was conducted using suspensions of PAO1 resting cells prepared from an actively growing culture at $\sim 10^{8} \mathrm{CFU}$ per millitre pelleted $\left(3000 \mathrm{~g}, 10 \mathrm{~min}, 20^{\circ} \mathrm{C}\right)$ and resuspended in half the volume of $20 \mathrm{~m} M \mathrm{NaPiB} \mathrm{pH} 6.0,150 \mathrm{~m} M \mathrm{NaCl}, 100 \mathrm{~m} M$ sorbitol. The sorbitol was added as an additional osmoprotectant to maintain cellular integrity. $100 \mu \mathrm{l}$ of this suspension was added to each well of a FluoroNunc 96-well plate together with $50 \mu \mathrm{l}$ of $40 \mu M \mathrm{NPN}$ and $50 \mu \mathrm{l}$ of the enzyme or peptide at the corresponding concentration, or just buffer for the negative control. Fluorescence was then immediately recorded in a Varioskan Flash microplate reader (Thermo Scientific) with an excitation wavelength of $350 \mathrm{~nm}$ and a fluorescence-detection wavelength of $420 \mathrm{~nm}$. Nondyed PAO1 cells treated with just buffer were also incorporated as a background measurement and EDTA was used as a positive control for OM permeabilization. Samples were also observed by fluorescence microscopy using an A filter cube (Leica) with a bandpass of $340-380 \mathrm{~nm}$.

\subsection{Fluorochrome labelling of Pae87}

An $N$-hydroxysuccinimidyl (NHS) ester of Alexa488 fluorochrome (NHS-Alexa488, Thermo Fisher) was used to fluorescently label purified Pae87. A labelling reaction was set up by incubating the dye together with the protein in a 1:5 (protein:dye) ratio in $20 \mathrm{~m} M \mathrm{NaPiB} \mathrm{pH} 7.4,100 \mathrm{~m} M \mathrm{NaCl}$ for $1 \mathrm{~h}$ at room temperature. At this $\mathrm{pH}, \mathrm{N}$-terminal amino groups would be more reactive than lateral chain amino groups, and therefore the dye would preferentially be incorporated at the N-terminus. The reaction was stopped by adding a $10 \%$ volume of $1 M$ Tris- $\mathrm{HCl} \mathrm{pH}$ 7.4. Free dye was then separated using a HiTrap Desalting FF $5 \mathrm{ml}$ column on an ÄKTA start liquid-chromatography system with $20 \mathrm{~m} M \mathrm{NaPiB}$ pH 7.4, $100 \mathrm{~m} M \mathrm{NaCl}$ as the mobile phase. The much smaller molecule of the dye was retained for longer within the column, while the dyed protein eluted earlier. The degree of labelling of each elution sample was calculated by estimating the protein and dye concentrations, measuring $A_{280}$ and $A_{495}$, respectively, and applying the Lambert-Beer law with the respective molar extinction coefficients. $A_{280}$ was corrected by a factor of 0.11 (corresponding to $A_{280} / A_{495}$ for the dye). Only samples with a labelling degree of $\simeq 1$ were used for experiments.

\subsection{Statistical analyses}

Statistical analyses were performed either in $R$ or using GraphPad InStat. Unless otherwise stated, quantitative differences between experimental conditions were analyzed to determine whether these differences were statistically significant under the assumption of a significance level $\alpha=0.05 \mathrm{using}$ ANOVA. The post hoc tests of Tukey or Dunnett were further used for multiple comparisons. Unless otherwise stated, all results presented are mean \pm standard deviation of at least three independent replicates. 


\section{Results}

\subsection{General analysis of the Muramidase family}

Pae87 contains a single predicted catalytic domain that occupies most of its full length (90.3\% coverage) and belongs to the recently described Muramidase family (PF11860, Pfam $E$-value $\left.1.1 \times 10^{-63}\right)$. The Muramidase family is thought to comprise $N$-acetylmuramidases (EC 3.2.1.17) which, as predicted by Pfam, are usually found in bacteria (mainly Proteobacteria) or bacteriophages from the Caudovirales family (Fig. 1a). Lytic enzymes in Pfam containing a Muramidase catalytic domain have architectures that range from a single Muramidase catalytic domain to Muramidase domains accompanied by different known CWBDs at either the $\mathrm{N}$ - or C-terminus (Fig. 1b). A sequence-similarity network (SSN) constructed with the set of Muramidase family representatives (Fig. 1c) contained in InterPro displays some similarity-based clustered groupings, such as those of proteins from Pseudomonas or some Alphaproteobacteria clusters, but in general it does not provide evidence of clear taxonomically relevant subfamilies. In a previously curated collection of phage lysin sequences named $\mathbb{S}^{\text {LYS }}$ (Vázquez et al., 2020), Muramidase domains accounted for $1.56 \%$ of the total predicted domains (Fig. 1d), mostly represented in phages that infect Gramnegative bacteria (Fig. 1e), among which the Muramidase family is not an uncommon catalytic domain. In the subset of $\mathbb{S}^{\text {LYS }}$ that comprises just those entries bearing at least a Muramidase catalytic domain, hereafter termed $\mathbb{S}^{\mathrm{MUR}}$, the length distribution contains two subpopulations (Fig. 1f) that can be related to the presence or absence of a CWBD, which is usually located at the N-terminus (Figs. $1 \mathrm{~g}$ and $1 \mathrm{~h}$ ). $54.3 \%$ of the lysins in $\mathbb{S}^{\mathrm{MUR}}$ contained two predicted domains (i.e. bear both a Muramidase catalytic domain and a CWBD) while the rest are thought to be monomodular, as is Pae87 itself. The

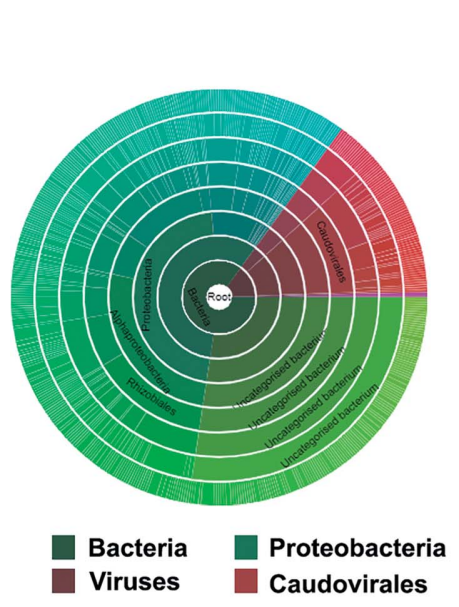

(a)

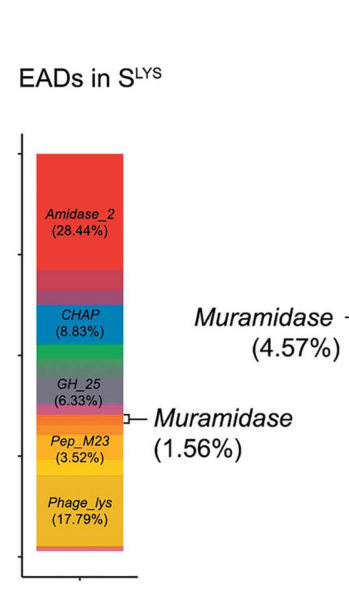

(d)

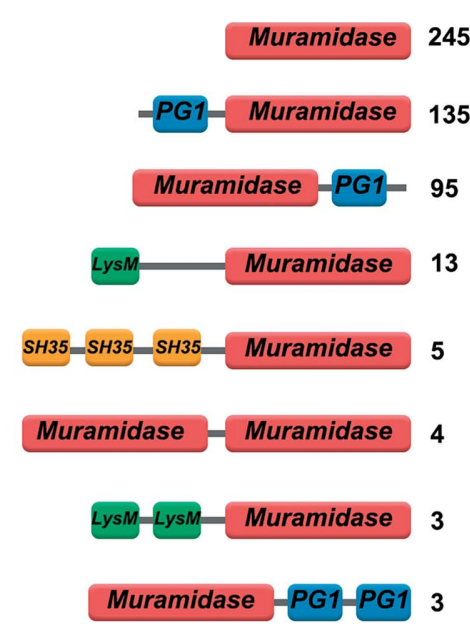

(b)

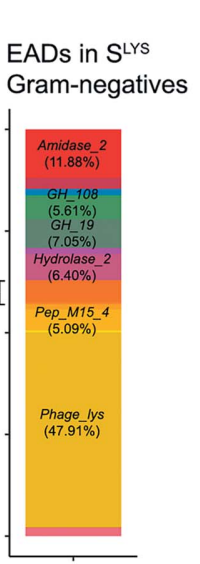

(e)

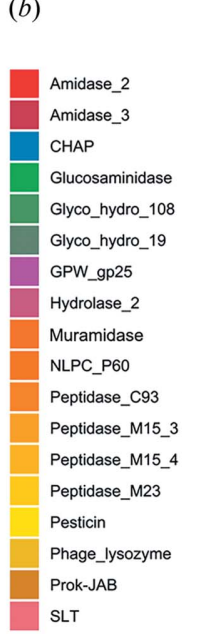

SLT

Figure 1

Overview of the Muramidase domain family. (a) Sunburst taxonomical representation of organisms bearing predicted Muramidase (PF11860)-containing proteins as taken from Pfam. (b) Main architectures found among Muramidase-containing proteins in Pfam. The numbers on the left are the number of representatives predicted to have such an architecture [PG1, blue boxes, PG_binding_1 (PF01471); SH35, orange boxes, SH3_5 (PF08460); LysM, green boxes, PF01476]. (c) SSN comprising the predicted Muramidase-bearing proteins in InterPro entry IPR024408 corresponding to the Muramidase family. $(d, e)$ Distribution of catalytic domains in the phage lysin curated database $\mathbb{S}^{L Y S}(d)$ or in the subset $\mathbb{S}^{L Y S}$ comprising only those lysins from phages that infect Gram-negative bacteria $(e)$. $(f)$ Length distribution of the Muramidase-containing lysins in $\mathbb{S}^{\text {LYS }}\left(\mathbb{S}^{\text {MUR }}\right)$. ( $(g)$ Distribution of the number of predicted domains in $\mathbb{S}^{\mathrm{MUR}}$. (h) Architectures found in $\mathbb{S}^{\mathrm{MUR}}$. (i) SSN of $\mathbb{S}^{\mathrm{MUR}}$. 
SSN of $\mathbb{S}^{\mathrm{LYS}}$ did not display a relevant taxonomical clustering (Fig. 1i), although the sample size was on the low side to be able to draw generalized conclusions.

\subsection{Three-dimensional structure of Pae87 and its substrate-binding region}

While evidence has previously been provided for the antimicrobial activity of Pae87 (Vázquez, Blanco-Gañán et al., 2021), in order to further elucidate the mechanism by which Pae87 interacts with susceptible bacterial cells we determined crystal structures of the protein with and without a bound peptidoglycan fragment.

The protein without and with PAO1 peptidoglycan was crystallized as described in Section 2. The best apoprotein crystal diffracted only to limited resolution and with somewhat streaky spots, but the best peptidoglycan-bound protein crystal diffracted X-rays to high resolution (Table 1) with cleaner spots. In the crystal packing, the peptidoglycan fragment contacts a neighbouring protein molecule; this may be the reason why the ligand-bound form crystallized in different conditions and in a different crystal form that diffracted $\mathrm{X}$-rays to higher resolution. A structure-prediction search in HHpred (Zimmermann et al., 2018) showed that AP3gp15, a Burkholderia AP3 phage endolysin that belongs to the Muramidase Pfam family, had the most similar sequence to Pae87 (50\% identity with 183 amino acids in the alignment). Therefore, the apoprotein was solved by molecular replacement using AP3gp15 (Maciejewska et al., 2017; PDB entry $5 \mathrm{~nm} 7)$ as a search model. The apo structure could only be refined to relatively high, but not unreasonable, $R$-factor values, while the peptidoglycan-bound Pae87 structure could be refined to more satisfactory values.

The crystallographic models of Pae87 comprise all 186 residues of the protein plus up to two residues from the N-terminal purification tag. The apo Pae87 and Pae87-peptidoglycan models have two and one molecules in the asymmetric unit, respectively. Molecule $B$ of the apo structure is less well ordered than molecule $A$, as shown by the average temperature factors ( 68 versus $52 \AA^{2}$, respectively). There is one amino acid with disallowed Ramachandran values in the apo structure: Gly32 in chain $B$. This residue has reasonable density and is near Leu76-Gly77 of chain $A$. Presumably, this contact pushes Gly32 into a somewhat unnatural conformation.

The root-mean-square deviations (r.m.s.d.s) between molecules $A$ and $B$ of the apo Pae87 structure and between molecules $A$ or $B$ of the apo structure and molecule $A$ of the Pae87-peptidoglycan structure are $0.5 \AA$ when $\mathrm{C}^{\alpha}$ atoms are superposed. Since the structures are almost identical, we mainly describe the Pae87-peptidoglycan complex structure and only add a few details specific to the apo Pae87 structure.

The crystallographic structure of Pae87 is almost identical to the C-terminal catalytic domain of the endolysin AP3gp15 (r.m.s.d. value of $1.1 \AA$ on 183 superposed $\mathrm{C}^{\alpha}$ atoms). Pae87 lacks the N-terminal CWBD present in AP3gp15 (Fig. 2a). The Pae87 structure shows the typical lysozyme-like $\alpha / \beta$ fold
Table 1

Crystallographic statistics of the Pae87 data sets.

Values in parentheses are for the highest resolution shell.

\begin{tabular}{|c|c|c|}
\hline & Pae87 & Pae87-peptidoglycan \\
\hline \multicolumn{3}{|l|}{ Data collection } \\
\hline Wavelength $(\AA)$ & 0.97926 & 0.97933 \\
\hline $\begin{array}{l}\text { Crystal-to-detector distance } \\
(\mathrm{mm})\end{array}$ & 488.7 & 212.5 \\
\hline Space group & $P 2_{1} 2_{1} 2_{1}$ & $P 2_{1} 2_{1} 2_{1}$ \\
\hline$a, b, c(\AA)$ & $59.25,68.09,93.10$ & $43.58,61.17,69.21$ \\
\hline Resolution range $(\AA)$ & $\begin{array}{l}59.25-2.50 \\
\quad(2.60-2.50)\end{array}$ & $\begin{array}{l}69.21-1.27 \\
\quad(1.29-1.27)\end{array}$ \\
\hline Total No. of reflections & $78046(8657)$ & $314568(14575)$ \\
\hline No. of unique reflections & $13640(1509)$ & $49510(2412)$ \\
\hline Completeness (\%) & $99.7(99.1)$ & $99.9(100.0)$ \\
\hline Multiplicity & $5.7(5.7)$ & $6.4(6.0)$ \\
\hline $\mathrm{CC}_{1 / 2}^{\dagger}$ & $0.991(0.860)$ & $1.000(0.903)$ \\
\hline$R_{\text {meas }}($ all $I+$ and $I-) \neq$ & $0.202(1.087)$ & $0.046(0.828)$ \\
\hline$\langle I / \sigma(I)\rangle$ & $6.0(2.1)$ & $17.6(2.0)$ \\
\hline Wilson $B$ factor $\left(\AA^{2}\right)$ & 31.8 & 13.9 \\
\hline \multicolumn{3}{|l|}{ Refinement } \\
\hline Resolution range $(\AA)$ & $55.02-2.50$ & $45.85-1.27$ \\
\hline Reflections used & 12941 & 46988 \\
\hline Reflections used for $R_{\text {free }}$ & 658 & 2454 \\
\hline$R$ factor $/ R_{\text {free }} \S$ & $0.222 / 0.278$ & $0.130 / 0.161$ \\
\hline \multicolumn{3}{|l|}{ Model statistics } \\
\hline \multicolumn{3}{|l|}{ No. of atoms } \\
\hline Protein & 2964 & 1506 \\
\hline Peptidoglycan & 0 & 50 \\
\hline CHES & 26 & 0 \\
\hline PEG & 13 & 14 \\
\hline Ethylene glycol & 0 & 52 \\
\hline Water & 99 & 121 \\
\hline \multicolumn{3}{|l|}{ Average temperature factor $\left(\AA^{2}\right)$} \\
\hline Protein & 60.3 & 20.9 \\
\hline Peptidoglycan & - & 50 \\
\hline CHES & 89.0 & - \\
\hline PEG & 73.4 & 42.2 \\
\hline Ethylene glycol & - & 44.4 \\
\hline Water & 41.4 & 35.1 \\
\hline \multicolumn{3}{|l|}{ Ramachandran statistics } \\
\hline Favoured & 96.5 & 97.8 \\
\hline Allowed & 99.7 & 100.0 \\
\hline Disallowed & 0.3 & 0.0 \\
\hline R.m.s.d., bond lengths $\dagger \dagger(\AA)$ & 0.0016 & 0.0073 \\
\hline R.m.s.d., angles $\dagger^{\dagger}\left({ }^{\circ}\right)$ & 1.1 & 1.4 \\
\hline Clashscore/percentile $+\neq$ & $1.17 / 100$ th & $2.76 / 97$ th \\
\hline MolProbity score/percentile§§ & $1.06 / 100$ th & $1.09 / 98$ th \\
\hline PDB code & $7 \mathrm{q} 4 \mathrm{~s}$ & $7 \mathrm{q} 4 \mathrm{t}$ \\
\hline
\end{tabular}

$\dagger \mathrm{CC}_{1 / 2}$ : correlation coefficient between intensity estimates from half data sets (Karplus $\&$ Diederichs, 2015). $\quad \neq R_{\text {meas }}=\sum_{h k l}\{N(h k l) /[N(h k l)-1]\}^{1 / 2} \sum_{i}\left|I_{i}(h k l)-\langle I(h k l)\rangle\right| /$ $\sum_{h k l} \sum_{i} I_{i}(h k l) . \quad \S R=\sum_{h k l}|| F_{\text {obs }}|-| F_{\text {calc }}|| / \sum_{h k l}\left|F_{\text {obs }}\right| . \quad$ - Calculated by MolProbity (Williams et al., 2018). †† Calculated by REFMAC5 (Evans \& Murshudov, 2013). 枺 The clashscore is the number of serious clashes per 1000 atoms, where the 100th percentile is the best among structures of comparable resolution and the 0th percentile is the worst. $\S \S$ The MolProbity score combines the clashscore, rotamer and Ramachandran evaluations into a single score that is normalized to be on the same scale as the X-ray resolution (http://molprobity.biochem.duke.edu/; Williams et al., 2018).

subdivided into two subdomains (Wohlkönig et al., 2010). The $\alpha$-lobe is composed of $\alpha$-helices 1 and 2 (Glu5-Glu29) and $\alpha 6-\alpha 12$ (Gly109-Lys186). In the $\alpha$-lobe subdomain, $\alpha 2$ is surrounded by the other $\alpha$-helices. The other subdomain is traditionally known as the $\beta$-lobe because it contained a $\beta$-sheet in the first known endolysin structures (as it does in Pae87). In Pae87, the $\beta$-lobe is formed by $\beta$-strand 1 (Ile43Glu46), $\alpha$-helices 3, 4 and 5 (Arg47-Ser99) and $\beta$-strands 2 and 3 (Ala100-Met108). The three $\beta$-strands form a small antiparallel $\beta$-sheet (Fig. 2c). Both subdomains form a cleft 


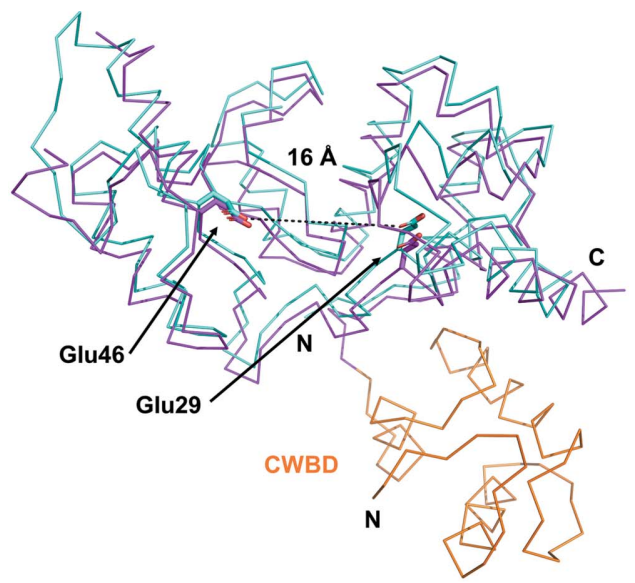

(a)

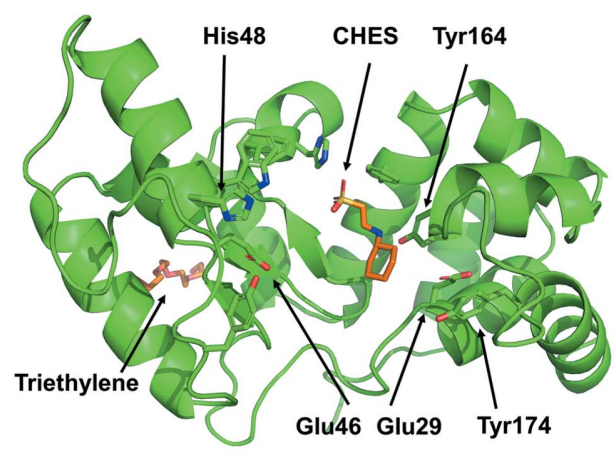

(b)

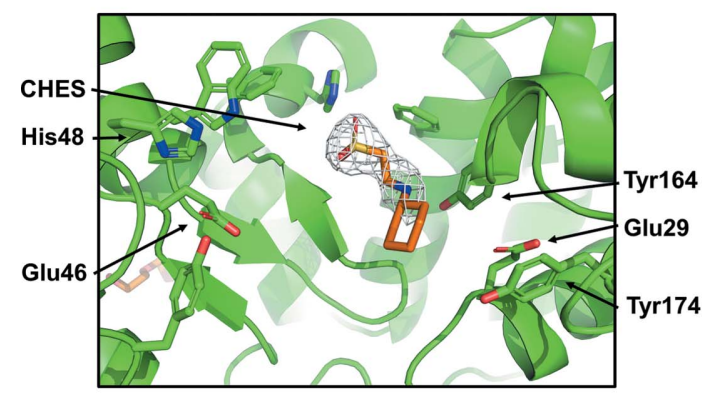

(d)

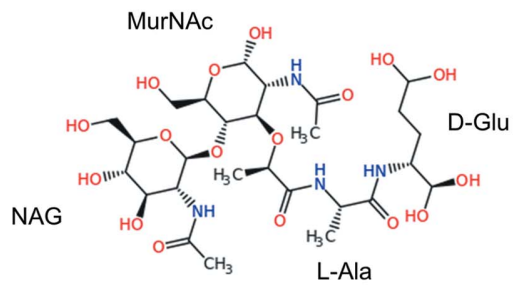

(f)

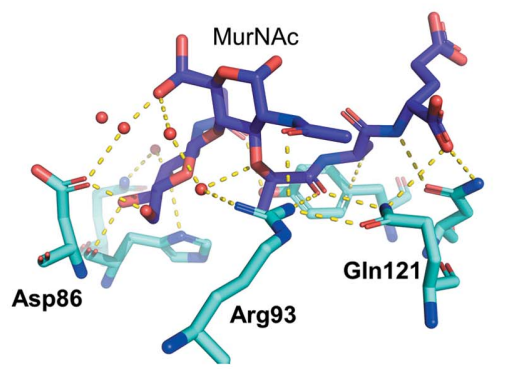

(h)

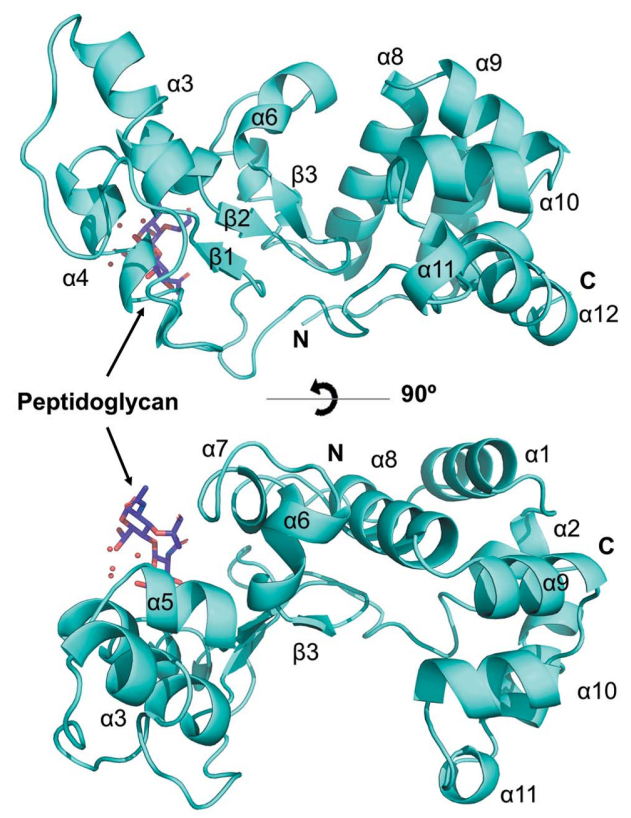

(c)

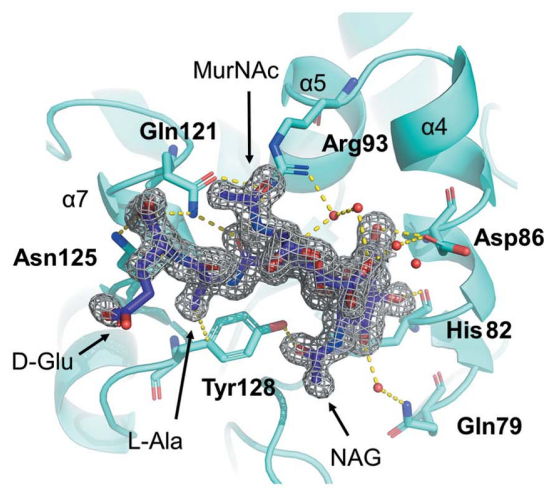

(e)

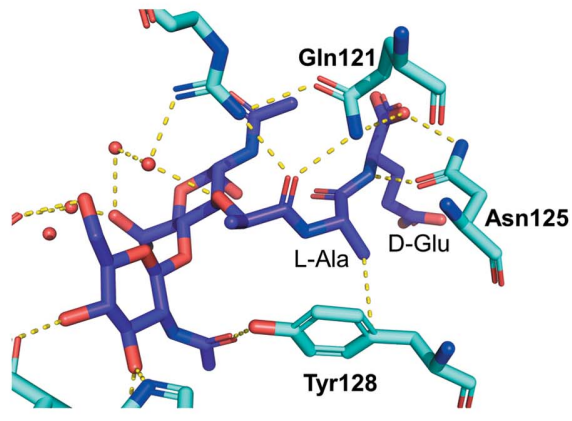

(g)

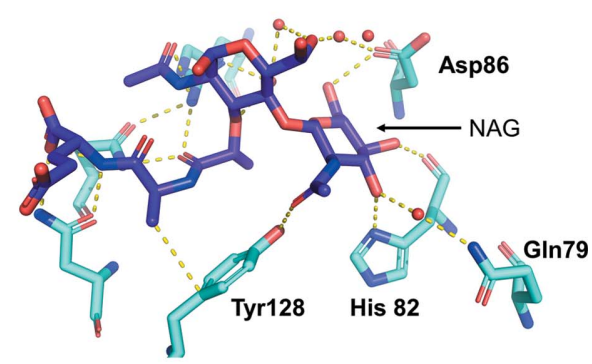

(i) which contains the putative catalytic residues Glu29 and Glu46.

In the apoprotein crystal, a CHES molecule was bound in the cleft next to the catalytic amino acids (Figs. $2 b$ and $2 d$ ). When Pae87 was crystallized together with the hydrolyzed peptidoglycan sacculi, we expected to see a fragment of it bound to the catalytic cleft. Surprisingly, the peptidoglycan fragment (comprised of linked NAG, MurNAc, L-alanine and D-glutamic acid units; Fig. $2 f$ ) was bound to $\alpha$ helices 4,5 and 7 on the opposite side to the putative catalytic pocket (Figs. $2 c$ and $2 e$ ), in the same place in which a polyethylene glycol fragment was modelled in the apo Pae87 struc-

Figure 2

Pae87 structure. (a) Superimposition of the catalytic domains of Pae87 (cyan, PDB entry 7q4t) on AP3gp15 (magenta, PDB entry 5nm7), with the AP3gp15 CWBD shown in orange. The putative catalytic residues Glu29 and Glu46 from Pae87 and Glu101 and Glu118 from AP3g015 are shown in stick representation. (b) Ribbon representation of the apo Pae87 model coloured green (PDB entry 7q4s). A CHES molecule is depicted as orange sticks and is flanked by the putative catalytic amino acids (Glu29 and Glu46) and several aromatic residues in stick representation. A triethylene glycol fragment molecule (orange sticks) is present at the back of the protein, in the same place as the peptidoglycan in $(e)$. (c) Side view (top) and top view (bottom) of the Pae87 protein model in ribbon representation (cyan). The peptidoglycan molecule is shown as violet sticks. $(d)$ Close-up view of the active site depicted as in $(b)$. The CHES molecule is represented with its $2 F_{\mathrm{o}}-F_{\mathrm{c}}$ electron-density map contoured at $1 \sigma$ (grey). (e) View of the peptidoglycan fragment depicted as in $(b)$ with its $2 F_{\mathrm{o}}-F_{\mathrm{c}}$ electron-density map contoured at $1 \sigma$ (grey), the binding amino acids (cyan, stick representation) and the water molecules (red spheres) taking part in the hydrogen-bond network (yellow dashed lines). $(f)$ Schematic representation in Fischer projection of the peptidoglycan fragment bound to Pae87. $(g, h, i)$ Close-up views of the peptidoglycan fragment components (D-Gly and L-Ala, MurNAc and NAG, respectively) depicted as in $(c)$. 
Pae87/1-168

ASV45028.1/1-167

AUV62708.1/1-173

QBP28097.1/1-168

$Q B Q 72232.1 / 1-167$

QBQ72917.1/1-172

QEM40954.1/1-167

QEM42170.1/1-173

QFR59726.1/1-170

YP_009006538.1/1-172

YP_009009383.1/1-168

YP_009616761.1/1-168

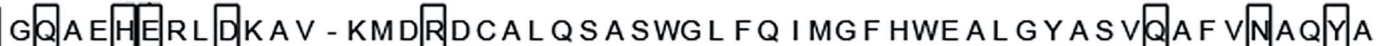
GADEHERLDT AA - KYDRDCALESA SWG SFQVMGYHWKALGYPTLQAFINDQYKK LNKEDD DM DRAAKE I DRTS ALEA A SWG AFQ I MGYHWKT CGYPVLQAFVNAQ YS GQSEHERLDKAV - SMDRDCALQSA SWGL FQ I MGF HWE AL GYKSVQAFVNAQ YA GS DEHTRL DQAA - KFDRDAALESA SWG AFQ IMGYHWKT L GYPSLQAF INAMYK - DE HG RL ARA T - S I DR DCAL SA A SWG AFQ I MGYHWKT L GYASL OEF INEMYT YSEQHAKL ARAV - K I DRDS AL ES CSWGMFQ I MG Y HWKL MG YPT LQAFVNNAMYA A LNKEDKDM DRAAKE I DRTS ALEAASWG AFQVMGYQWKACGYPALQAF INAQ YS ESAQHGRLORAS - AL NRDVAL A S A SWGL GQVLGENWKDLGYKSLOSF VNAMYKK YSEQHKKL DRAA - EL NRTVALES CSWGL GQVMGYHWKSLGYTNL QEF INCMYYK GAAEHIRLNTAV-MIDRECALQSA SWGLFQ IMGF HWKAL GFESVQQF INAQ YKK FSEQHGKLLRAV - K I DBE C A L Q S C SWGMF Q L MG F NYKL CG Y A T VQA F V NAMUK

\section{Pae87/1-168}

AIP84309.1/1-173

AKA61137.1/1-171

AKU43570.1/1-167

ATW66849.1/1-174

AVP40008.1/1-172

AYP28388.1/1-174

AZF87852.1/1-172

NP_944336.1/1-172

NP_958132.2/1-174

QBI84119.1/1-172

QBQ78849.1/1-173

QIG62158.1/1-173

YP_001294864.1/1-173

YP 001956952.1/1-171

YP_004306400.1/1-171

YP_004306445.1/1-173

YP_004306871.1/1-171

YP 007349105.1/1-174

YP_009595839.1/1-175

YP 009609055.1/1-171

YP_009616095.1/1-173

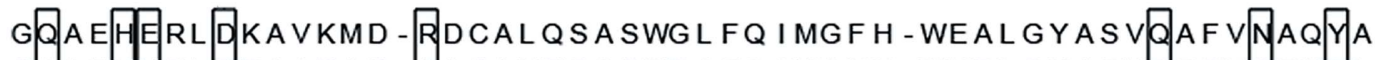
GAAEYYYRL DT AARID-AA SAYESASWGAFQ IMAYH - WKRLGYASVDEFVSSRMEL $D A A E Y A R L A S A S Q I S-G A C A L E A T$ TWG AFQ IMGFH-WKALGYPDVFAFVDAMIKV R-- - YDT LL AMVRLD-YDAGFASASYGRFQ IMGEN - AGMCGYASSMAFAEAMAR GDAEL ERLOGA I ALD- EDCAYESASYGLFQIMGFN - CQICGYSNAKEMFTDFLT KAGEHTRLAQA I A I D-RS CAL ASA SWGLFQ IMGYQ-SERIGYPSVDAFVQAMOS - TNERARL EKAVS ID-AECAYSSASYGLFQIMGFNGVKVCGYPTAKAFYMDMLLN GTAEHQRLANARQ I D-DT AAL ESA SWG AFQ IMGFH - WQRLGY I SVQAFAEAMG R GAGEMYVFF RVAAVTGDEVAAQCCSWG LGQVMGAN - YAT CGFT NAYGLMFASAL GAGEMYRF RVVAAVTGDEVAAQCCSWGLGQVMGAN - YATCGFT NAYGLMIFASAL GTAEHQRLANARQ I D-DT AAL ES A SWG AFQ IMGFH - WQRL GY I SVQAFAESMGR GDAEL ERL HGA I A ID-KDCAYESASYGLFQIMGFN-CVICGYANAEQMFNDFLT GDAEL DRL HAA INID- EDCAYESASYGLFQIMGFN-CQVCGYANAKEMFNDFLT GAGEMYRF ERVAAVTG DEVAAQCCSWG LGQVMGAN - YATCGF TNAYGLMFASAL KSAEYTTRLEQAEKID-EVSALESCSWGAYQIMGYH-WKLLGFGSVHDFLAAKMKKE GAAEYARLASASQIS-AACALEAASWGAFQIMGFH -WNALGYPDVFAFV DAMKII GSAEYYVL DTAARIH-AASAYESA SWG AFQVMGYH -WKRLGYASI DEFVARMET KAAEYVRLAATAERID-TDASHESASWGAFQIMGYH-WQALDYSSIADFVACMOR -TNERARL EKAVT I D-AECAYASASYGLFQIMGFNGVKVCGYPTAKAFY IDMLLN INDSMEKLAEAACKD-P DAAFKSVS IGKFQVMGAY - YKELGYSHPIIEMLMDARN GEKEMVRL ENAMT IN-KDAAL ESA SWGAYQIMGFH - WLLLGFKSVQEYVQFTSV GDAELLRL HGA IA I D- UDCAYESASYGLFQ IMGFN - CV I CGYANAEQMFNDFLᄂ

(a)

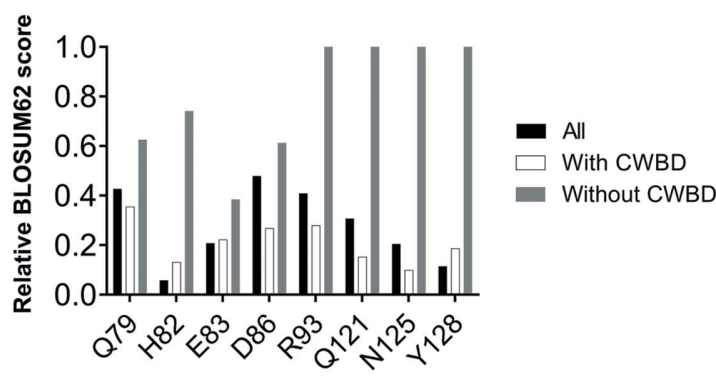

(b)

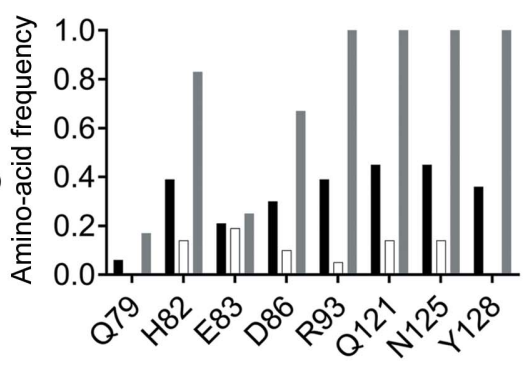

(c)

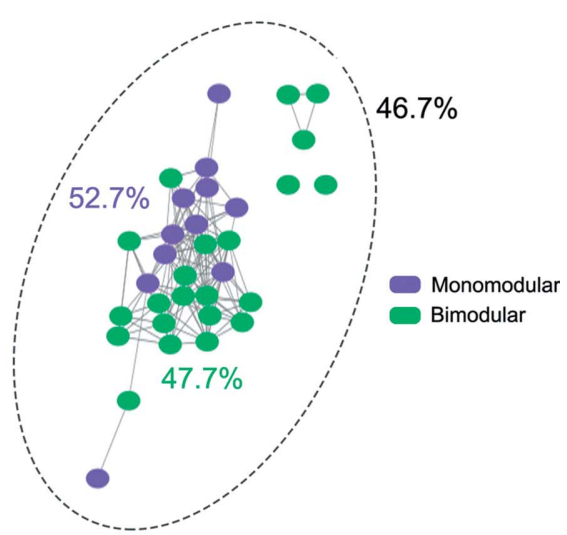

(d)

Figure 3

Multiple sequence alignments (MSAs) of the peptidoglycan-binding site of lysins in $\mathbb{S}^{\mathrm{MUR}}$. (a) MSA of the peptidoglycan-binding site of Muramidase family lysins grouped by the presence or absence of an N-terminal CWBD. The residue coordinates indicated at the top are those of Pae87. ( $b, c$ ) Residue-conservation metrics for the residues putatively involved in contacts with peptidoglycan at the peptidoglycan-binding site according to the Pae87 3D model. The relative BLOSUM62 score is shown in $(b)$ and the relative frequency of the Pae87 residue across the MSAs in each respective position is shown in $(c)$. (d) SSN of the 33 sequences of the MSAs distinguished by the presence or absence of a CWBD. Percentages are average identities for each group. The entries AKU43570.1 and YP_009595839.1 were reclassified as CWBD-bearing lysins since they contained an unidentified $\mathrm{N}$-terminal end that could probably be an as yet undescribed CWBD. 
ture (Fig. 2b). This fragment was mainly bound by a hydrogenbond network formed by Gln79, His82 and Asp86 ( $\alpha 4)$, Arg93 $(\alpha 5)$, Gln121, Asn125 and Tyr128 ( $\alpha 7)$ (Figs. $2 g-2 i)$. Tyr128 probably establishes a $\mathrm{CH}-\pi$ interaction with the L-Ala methyl group. The most important binding residues would be Arg93, Gln121, Asn125 and Tyr128, because they each make more than one bond to the ligand.
A multiple sequence alignment (MSA) analysis of the amino acids composing the peptidoglycan-binding site using $\mathbb{S}^{\text {MUR }}$ (removing two low-coverage examples: YP_009639957.1 and YP_337984) revealed that the residues relevant for peptidoglycan binding were much more conserved in those lysins bearing a single Muramidase catalytic domain than in those which had an additional CWBD (Fig. 3a). Specifically, in
E29

- VASVKAVTKVESRGSGFLLSGVPKI - - - LFERHWMF - LACVRAVNEVESRGVGFLDDGRPKI - - - LFERHVMY - LAAVRAVNEVESKGAGFLPDGRPVI - - - LYERH I MY - - RNIKAVTQVEAPGSG - FQAGLPKI - - LPEPHRFS - VAAVKAVSSVESSGNGFLADGRPKI - - - LFERHIFK ELASMKAVNQVESRGTGFTKTGKIKT - - LFERHIMY - VPALRAFAEVESNGSGF I DGKRPKV - - QYEPHVMY - VAAVRTVNEVESRGSGFLPDGRPVI - - - LFERHVMY ELAA I KAVNQVEARGSGYTKTGDLKM - - LFERHIMF - LAT I YAVNQVESNGQGFLGNGKPA I - - LFER H IMY - VPAFQA I I QVETTMGSGFLPDGRPRI - - - L LERHKVW - TPALQA I I QVEETTGSGFLPDGRPR I - - - L LER R H KVW - LAT I YAVNQVESNGQGFLGNGKPA I - - - LFERH I MY - VASVKAVTKVESRGSGFLLSGVPKI - - - LFERHWMF - VPAVKAVAEVEANGMGFLPDGRPKI - - - LFERHIFR - EAA I KAVAAVESNGNGFTVDAGMKVPKVLFERHVMY - LAS I KAVNQVESRGTGFTKSGKIKT - - LFER H I MY - VPA I KAVTKVEAPVGGFQPTGEPT I - - L L ERHQMY - VPALRAFAEVESNGSGF I DGKRPKV - - QYEPHVMY - - AALRAVMDVECKGRGFNDDGTPVI - - LFERHKFY - LASVKAVNQVESRGTGFTKSGKIKT - - LFERHIMY - VPAFQA I I QVETT TGSGFLPDGRPR I - - - LLERHKVW - LPAVKAVKEVEAPAGGFLPDGRVT I - - - LY E R H VMF - LAAVRAVNEVESRGAGFLPDGRPVI - - - LFERHVMY - PACVRAVTEVESRGSGFLADGRPVI - - LFERHVMY - VAS I RAVNEVESRGEGF I VDGRPA I - - LFER HVFY ELAA I KAVNQVEARGSGYTKSGDLKM - - LFER H I MF - LAAMKA I VEVECKGSGFNSDGTPVI - - LYERHKFY EPACVKAVTKVESRGSGFLPSGEPV I - - L F ER HWMY - - RRIKAVASVESGGSGWF DSGLPKI - - L Y ER R HFY - TAALMAVNDVESKGTGFLANGRIA I - - L Y E R H I MY - LAS I KAVNQVESRGTGFTKSGKIKT - - LFER H I MY EVAA I KA I ASVETKGSAWI TPGVPQ I - - L YERH I MA
YP_009616095. YP_009616761.1/1-168

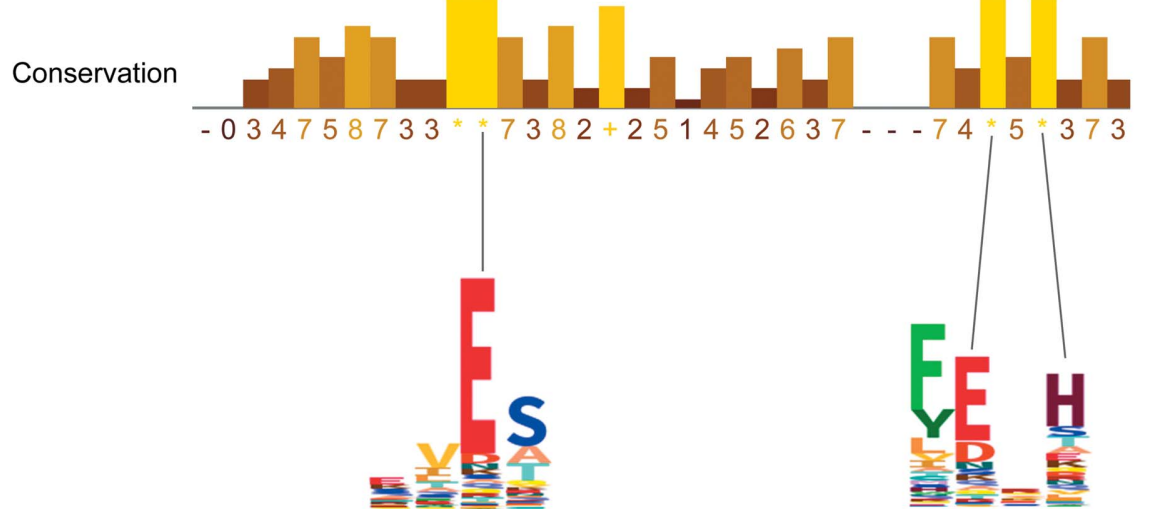

Y178

KKNNYDVKLAEAYQSFK A I NLYDVKLDRAYAKY $A E N L Y D V K L E R A F D R Y-$ AKQNYHGK I AAAV - . AKNSYHTKMSSAYKQF AKNQYDTKLAAAYKSF KKNAYDDKLAKAYKKY KDNLYDVKLARTFARYQ $A V D G Y D K K L A A A Y A S F$ KRNLYDTKL ARAYEQH A I NQYDTKL DKAYI - - A I NQYDTKL SDAYTTL KRNLYDTKLARAYEQH KKNNYDVKLAEAYQSFK AKNNYDSRLAAANKR - SKFNYDKKMALEYAK - AQNQYYDTKLAAAYKSF AKNQYDVKLEKAYAE - KKNAYDDKLAKAYKKY AENKYDTKLAAAYKK - AKNQYDTKLAAAYKSF A I NRYDTKLAEAYTA - RQNNYDVKLASAYTKF AENLYDVKLERAFDRY ARNLYDAKLAQAYERY ARNL YDAKL AQAYTKY AADGYDKKLAAAYASF RKNQYDTKLSAAYKKF A I NKYDTKLAAAYASF EKNNYYH I KL ANAMKKF KENQYDTKLAAAYKQY $A Q N Q Y D T K L A A A Y K S F$ KINSIYDQLAVAYES - -

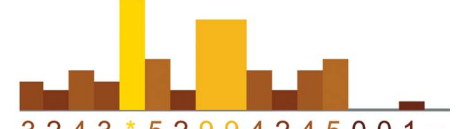
$3243 * 52994245001-$

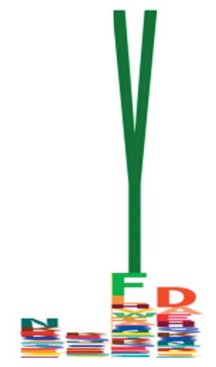

Figure 4

MSA of Muramidase family domains in $\mathbb{S}^{\text {MUR }}$ showing the conservation of residues facing the catalytic pocket. Selected segments of the Pfam HMM logo of the family are displayed at the bottom. Relevant positions are connected to their respective columns in the MSA. 
the case of Tyr128 and Arg93, together with Gln121 and Asn125, both the amino-acid frequency and the relative BLOSUM62 score (which is a similarity metric) were close to the maximum value (1.0) for the non-CWBD-bearing entries (Figs. $3 b$ and $3 c$ ). For the bimodular lysins of the Muramidase family, the scores were below 0.4 and 0.2 for Tyr128 and Arg93, respectively. The SSN in Fig. 3(d) shows that no sequence-identity bias was introduced by the classification since the average identity percentage both within each subgroup and in the whole collection of sequences was around $46-52 \%$ and there was no apparent clustering.

\subsection{Analysis of the Pae87 catalytic centre}

A single glutamic acid has been pointed out to be involved in catalysis in AP3gp15 (Maciejewska et al., 2017). A corresponding Glu residue is also conserved in the Pae87 structure (Glu29; Fig. 4) and in all of the 32 Muramidase sequence examples in the curated $\mathbb{S}^{\text {MUR }}$. Glu29 is located within a deep cleft between the subdomains of the Pae87 structure (Fig. 2a), and thus this is considered to be the catalytic site. This glutamic acid residue is conserved in many endolysins, such as AP3gp15 (PDB entry 5nm7; Maciejewska et al., 2017), hen egg-white lysozyme (HEWL; PDB entry 4hpi; Ogata et al.,
2013) and the peptidoglycan hydrolase Auto (PDB entry 3fi7; Bublitz et al., 2009). Most lysozymes have a catalytic site formed by two residues, a general acid and a general base, which are normally acidic amino acids (Glu or Asp). The C-terminal region of the central $\alpha$-helix $(\alpha 2)$ of the lysozymelike $\alpha / \beta$ fold typically contains a conserved catalytic glutamate, but the general base is not well conserved and does not exist in some lysozymes. In HEWL it is located $5 \AA$ away at the other side of the cleft, and in the peptidoglycan hydrolase Auto the general base is $14 \AA$ away in the antiparallel $\beta$-sheet (Bublitz et al., 2009).

Recently published results regarding the bimodular lysin LysT84, which contains an N-terminal PG_binding_1 (PF01471) CWBD plus a Muramidase enzymatically active domain, as AP3gp15 does, have pointed out the involvement of a second Glu residue in the catalytic activity (Love et al., 2022). Indeed, this second Glu residue (Glu46 in Pae87) was found to be perfectly conserved across all of the Muramidase examples considered in this work (Fig. 4). This perfect conservation strongly suggests that it plays a relevant role, possibly as the general base, located at a distance of $16 \AA$ from Glu29 (Fig. 2a), in a similar fashion as described for the Auto peptidoglycan hydrolase.

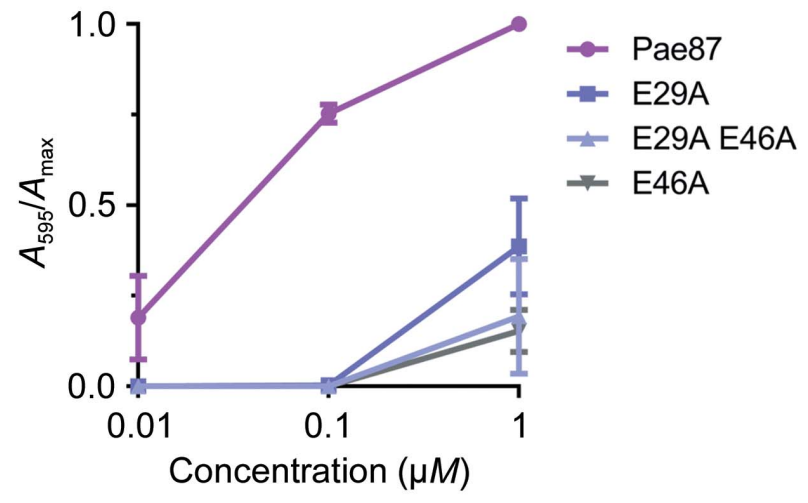

(a)
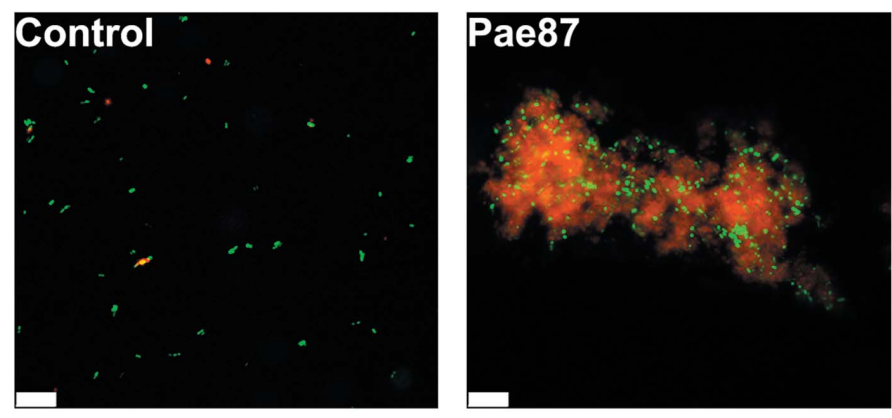

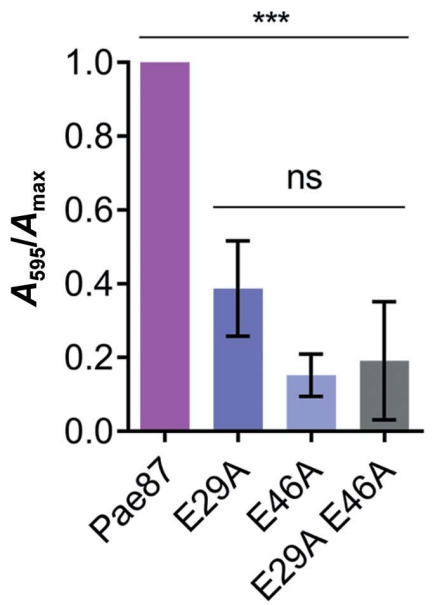

(b)

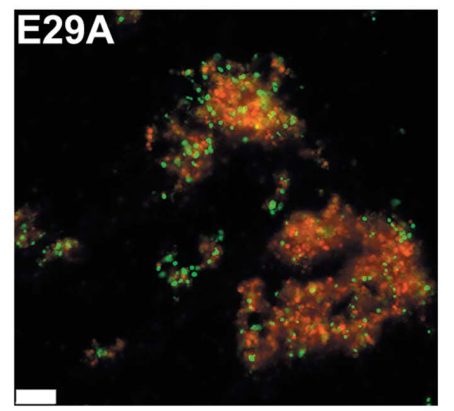

(d)

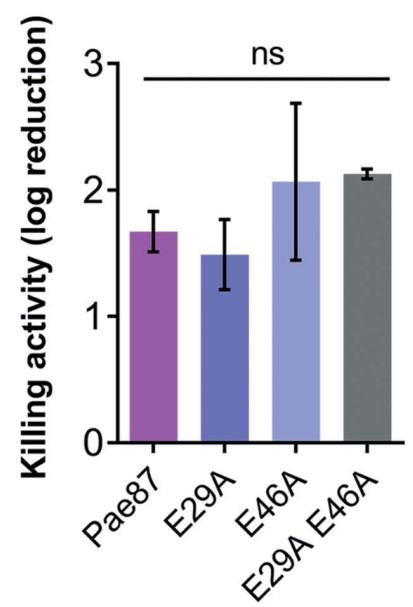

(c)

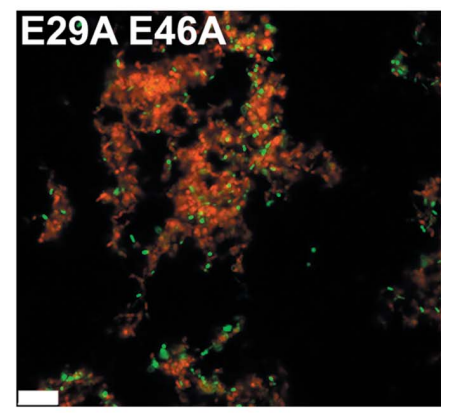

Figure 5

Activities of wild-type Pae87 and of mutants in which conserved catalytic site glutamic acid residues were mutated. (a) Muralytic activity of Pae87 and its mutants on RBB-labelled purified PAO1 cell walls at different concentrations. (b) Comparison of muralytic activities at the maximum concentration tested $(1 \mu M) .(c)$ Bactericidal activity of $10 \mu M$ Pae87 and its mutants against bacterial suspensions of PAO1 $\left(2 \mathrm{~h}, 37^{\circ} \mathrm{C}\right)$. (d) Fluorescence microscopy of some of the treated suspensions in $(c)$ dyed with SYTO9 and propidium iodide. White bars at the lower left corner indicate the scale $(10 \mu \mathrm{m})$. One-way ANOVA was used in $(b)$ and $(c)$ followed by a Tukey post-test to perform an all-against-all multiple comparison (ns, non-significant difference; ***, $p \leq$ $0.01)$. 
Other conserved residues facing the catalytic cleft were His48 and Tyr174, which therefore could also play a significant role in the integrity and function of the catalytic pocket. The aforementioned residues also presented high conservation levels within the Pfam hidden Markov models (HMMs) of the family, judging by the HMM logos (Fig. 4), strengthening their presumptive functional role. In the apo Pae87 structure, electron density for a CHES buffer molecule was found inside the putative catalytic cleft between the putative catalytic glutamates (Figs. $2 b$ and $2 d$ ). Buffer molecules have also been found in the catalytic clefts of other enzymes, such as the lytic $\mathrm{CHAP}_{\mathrm{K}}$ domain of the endolysin LysK from Staphylococcus bacteriophage $\mathrm{K}$ or chitinase $\mathrm{C}$ (ChiC) from Streptomyces griseus HUT6037 (Sanz-Gaitero et al., 2014; Kezuka et al., 2006). They may mimic the enzymatic substrate or product, and thus their position reinforces the idea that Glu29 and Glu46 possess catalytic activity in Pae87.

A mutational analysis was conducted to confirm the implications of both Glu29 and Glu46. Pae87 single mutants (E29A and E46A) and a double mutant (E29A/E46A) were constructed and their muralytic and bactericidal activities were tested (Fig. 5). Both residues, Glu29 and Glu46, were deemed to be relevant for the catalytic degradation of PAO1 peptidoglycan since all of the mutants displayed a remarkable decrease in their cell-wall solubilization ability when compared with wild-type Pae87 (Figs. $5 a$ and $5 b$ ). At the

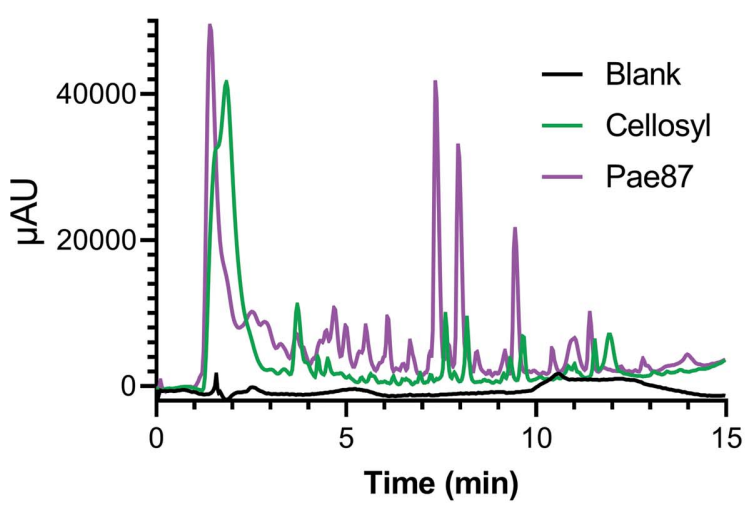

(a)

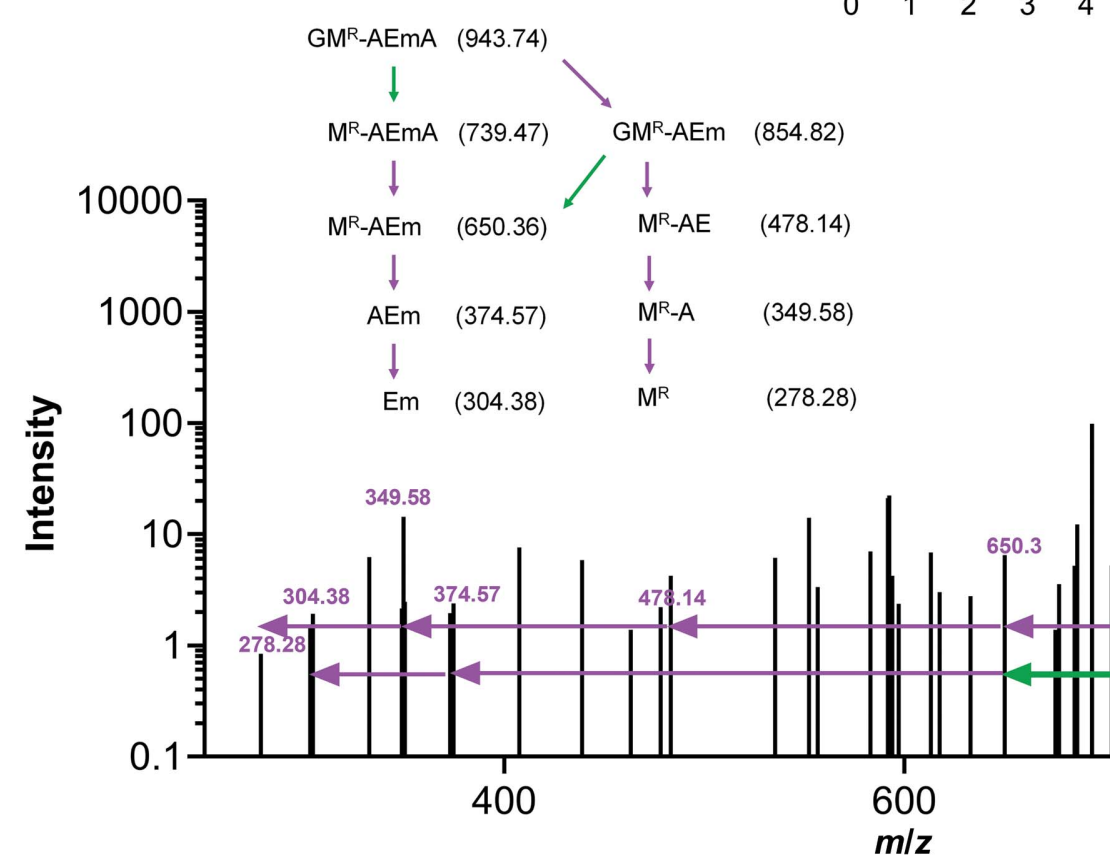

(c)

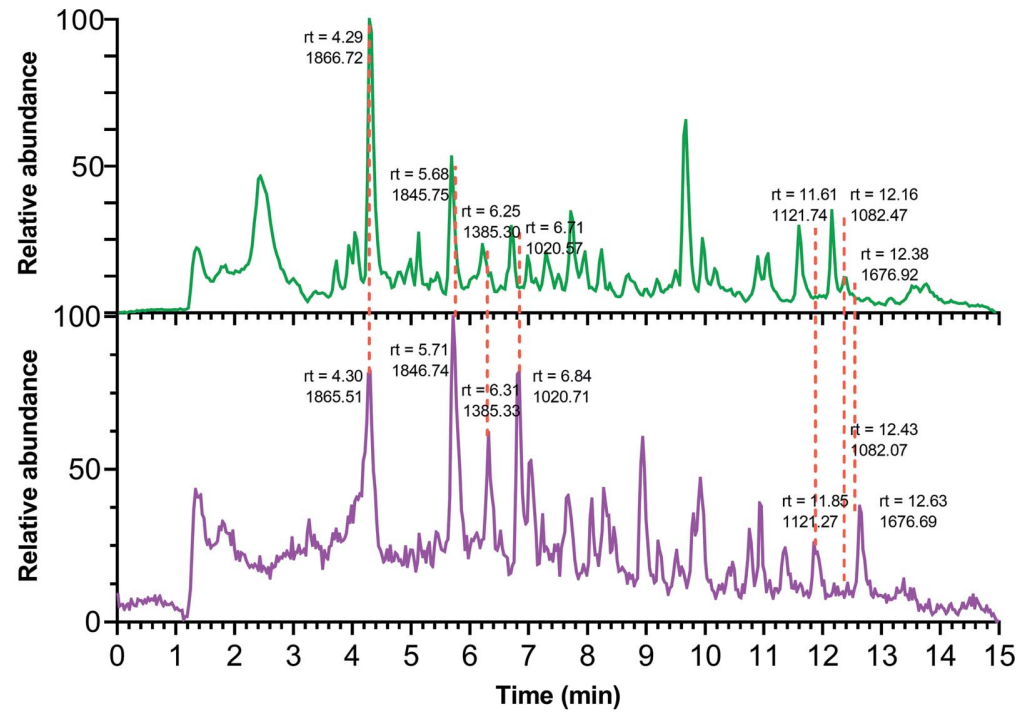

(b)

Figure 6

Analysis of the degradation products of Pae87 activity on P. aeruginosa PAO1 peptidoglycan. (a) UV (204 nm) chromatograms of PAO1 peptidoglycan solubilized with either cellosyl or Pae87 and of the soluble fraction of an untreated sample. (b) Liquid chromatography-mass spectrometry chromatograms of the degradation products of cellosyl (green) and Pae87 (purple) activities on PAO1 peptidoglycan. The retention time (rt) and a representative monoisotopic mass value are shown for selected peaks. $(c)$ CID spectrum of the $\mathrm{rt}=4.30$ min peak of the Pae87 peptidoglycan degradation. Selected $\mathrm{m} / \mathrm{z}$ values are displayed. The dissociation of a $\mathrm{GM}^{\mathrm{R}}$-AEmA fragment is presented. G, nonreduced NAG; $\mathrm{M}^{\mathrm{R}}$, reduced MurNAc; A, alanine; E, glutamic acid; m, meso-diaminopimelic acid. 
maximum nonsaturating concentration $(0.1 \mu M)$, Pae87 retained about $75 \%$ of its maximum detected activity, while the mutants displayed no peptidoglycan solubilization activity. On the other hand, there were no significant differences in the observed bactericidal activity against PAO1 between Pae87 and its mutants or in the fluorescence microscopy images (Figs. $5 c$ and $5 d$ ).

Regarding the catalytic activity of Pae87, the disaccharide found in the soluble product bound to the crystallized protein (NAG-MurNAc rather than MurNAc-NAG) already indicates lysozyme activity, as described in the literature for this family. Analysis of the degradation products by RP-HPLC-MS firstly showed that both Pae87 and the positive control (the lysozyme cellosyl; Rau et al., 2001) mobilized an array of soluble compounds, in contrast to the untreated blank (Fig. 6a). When comparing the mass-spectrometry chromatograms of cellosyl and Pae87, a coincidental pattern for the main degradation peaks was found (Fig. 6b). This observation supports the catalytic nature of Pae87 as a muramidase. Moreover, the CID spectrum of one of the main peaks of Pae87-degraded peptidoglycan (that at $4.3 \mathrm{~min}$ ) presents peaks that are compatible with the loss of a nonreduced NAG ( -203.078 mass units) from a NAG-MurNAc-Ala-Glu-mDAP-Ala or a NAG-MurNAc-Ala-Glu-mDAP fragment (Fig. 6c). Conversely, there is no evidence that is coherently compatible with the loss of a reduced NAG $(-223.106)$.

\subsection{The noncatalytic activity of Pae87 and P87}

The hypothesis that Pae87 displays a noncatalytic bactericidal activity has previously been proposed (Vázquez, BlancoGañán et al., 2021), and it is consistent with the results presented in Fig. 5. A closer examination of the physicochemical profile of Pae87 allowed us to postulate a specific 29-amino-acid peptide, named P87, which would make up a C-terminal AMP-like region (Fig. 7). The definition of P87 was based on the concurrent maximization of net charge and HM that takes place in the coordinates of Pae87. To measure these properties simultaneously, a score variable was calculated as explained in Section 2. The P87 sequence contains seven positively charged Arg or Lys residues (and a negatively charged Glu), interspersed with mostly nonpolar residues. This structure, together with the fact that it forms three $\alpha$-helices within the Pae87 structure (Fig. 7), suggests that it may form amphipathic helices with membrane-interaction potential.

The antimicrobial properties of the synthetic peptide P87 were analyzed. Circular-dichroism spectra in the presence of increasing concentrations of TFE provided evidence for the ability of P87 to form amphipathic helices in the presence of biological membranes (Fig. 8a). P87 presented a disordered conformation in aqueous solution; however, beginning at $20 \%$ TFE it started to shift towards an $\alpha$-helical conformation, as shown by the typical peaks at approximately 222 and $208 \mathrm{~nm}$. An acute lytic effect of P87 on P. aeruginosa PAO1 was observed, in contrast to the lack of generalized lysis when using the full Pae87 protein (Fig. $8 b$ ). An $\mathrm{OD}_{600}$ peak at $t_{0}$ is observed for both Pae87 and P87 above the turbidity value of the control. This has been noted previously when testing surface-active proteins on intact bacterial suspensions (Vázquez, Blanco-Gañán et al., 2021), and we speculate that it is due to the cell aggregation induced by the treatment. The subsequent decrease in turbidity should then be due to cell disintegration (which is much more acute in the case of the highly lytic peptide) and/or the formation of larger cell-debris aggregates that leave regions of the bottom of the well free of cells, thus potentially leading to a lower OD value. P87 was also able to kill PAO1 and other bacteria with a similar range and magnitude to Pae87 (Fig. 8c). The antimicrobial capacity of P87 is thus considered to be proven, and therefore a role in the Pae87-bacterial surface interaction can be proposed with supporting evidence.

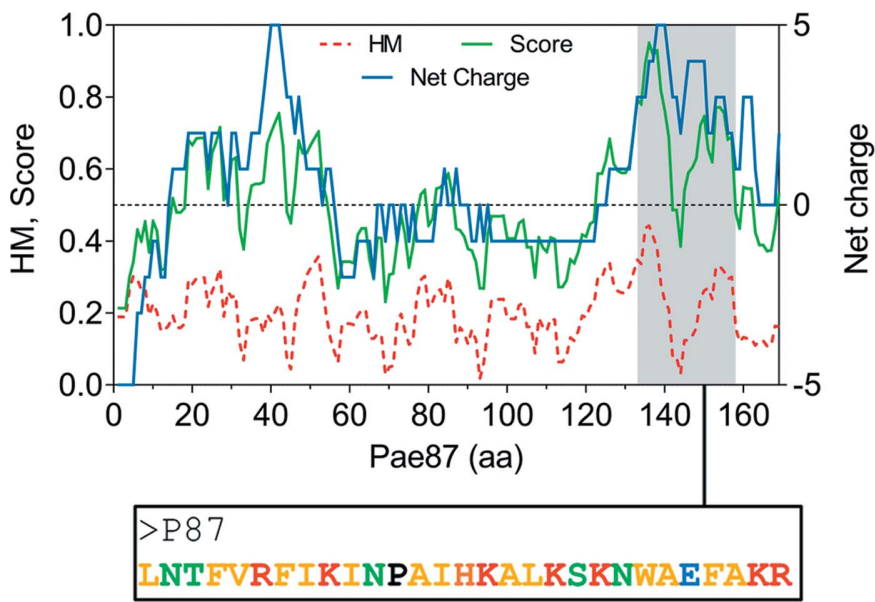

(a)

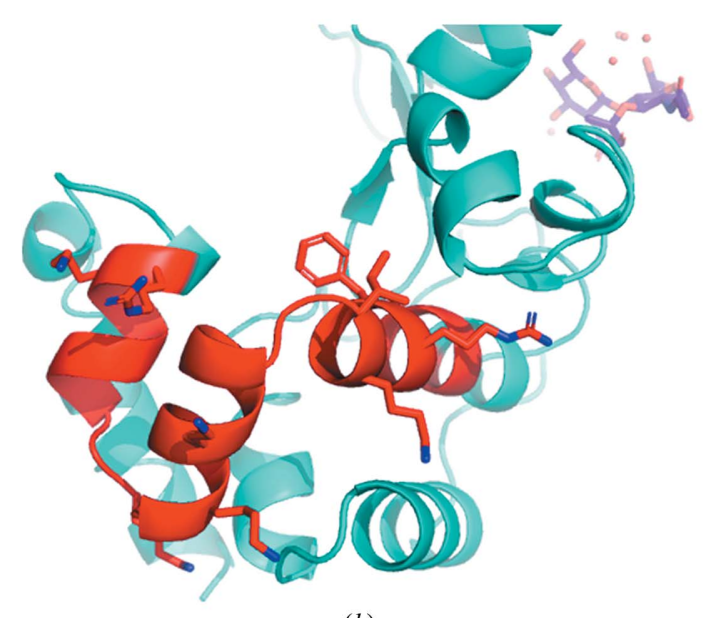

(b)

Figure 7

Definition and localization of the P87 peptide within Pae87. (a) Physicochemical profile of Pae87, depicting the HM and net charge in each of the 11-amino-acid windows of the protein, as well as the score used to measure both of these properties. The P87 sequence is also shown colour-coded with respect to the properties of each residue (yellow, nonpolar; green, polar without charge; red, positively charged; black, proline; blue, negatively charged). (b) 3D model of Pae87 with peptide P87 highlighted in red. 


\subsection{Antimicrobial mechanism of Pae87 and P87}

Since the OM has traditionally been labelled as the main obstacle when attacking Gram-negative bacteria from without, a fluorescent probe was used to detect OM permeabilization induced by Pae87 and its derivatives (i.e. the noncatalytic mutants and the antimicrobial peptide P87). NPN is a hydrophobic molecule that fluoresces when it reaches the phospholipid layer of the inner membrane upon OM permeabilization. Typically, NPN uptake kinetics are recorded with short incubation times (with a maximum of $10 \mathrm{~min}$ and a minimum of $3 \mathrm{~min}$; Loh et al., 1984; Helander \& MattilaSandholm, 2000). However, we extended the incubation period to $2 \mathrm{~h}$ due to the increasing fluorescence values of Pae87 kinetics over such a time (Fig. 9a). In the average kinetic estimations shown in Fig. 9(a), two different tendencies can be observed: the 'canonical' OM permeabilization peak induced by P87 in the first minutes after peptide addition and the slow but steady increase in fluorescence for Pae87 and its mutants. For the sake of statistical comparison, the average values of the NPN fluorescence reached 5 min after reagent addition were considered (Fig. 9b). Over such a short period the NPN fluorescence of Pae87 and its noncatalytic mutants is still low, although for Pae87 it is significantly different from the control. The fluorescence induced by P87 was greater than that obtained for the EDTA positive control. Fluorescence microscopy images at the end of incubation visually confirmed the damage to the $\mathrm{OM}$ for all of the compounds tested (Fig. 9a).

A fluorescent version of Pae87 labelled with Alexa488 was used in combination with PI, a DNA-intercalating fluorescent probe. In this way, the temporal localization of Pae87 and its effect on a suspension of PAO1 cells were traced (Fig. 10). At $t_{0}$ Pae87 had already begun binding to the bacterial surface, as green fluorescence rims were observed around the $P$. aeruginosa cells (Fig. 10a). Over time, the Pae87 molecules bound to the cell walls promoted increasing aggregation among close bacteria. According to visual estimations of the areas of these aggregates, their maximum size was reached after approximately 30-60 min under the assay conditions (Fig. 10b). Also, at $t_{0}$ a discrete intracellular spot of red fluorescence (PI) was observed. Subsequently, the PI fluorescence underwent a gradual diffusion concomitant to the aggregation. At $60 \mathrm{~min}$ of incubation, red fluorescence appeared as a halo around the aggregates, with a distorted bacterial morphology.

Several incubation conditions were examined to check their importance in Pae87 and P87 killing activity (Fig. 11). The

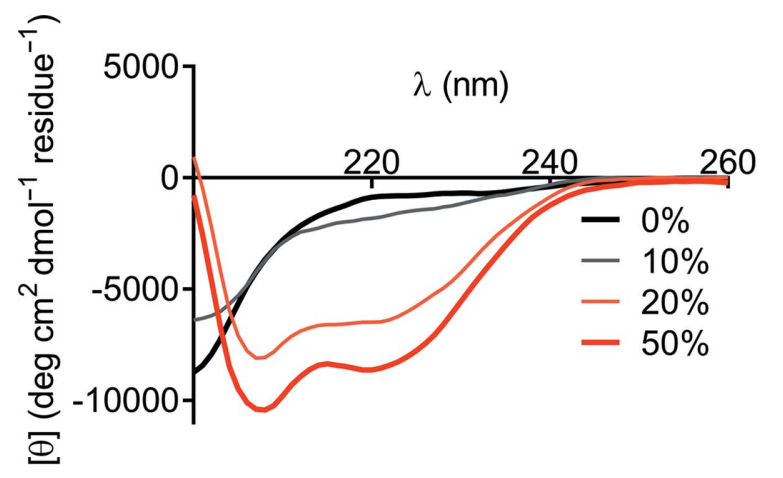

(a)

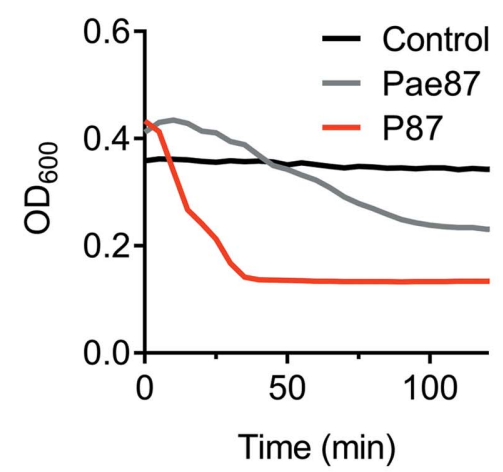

(b)

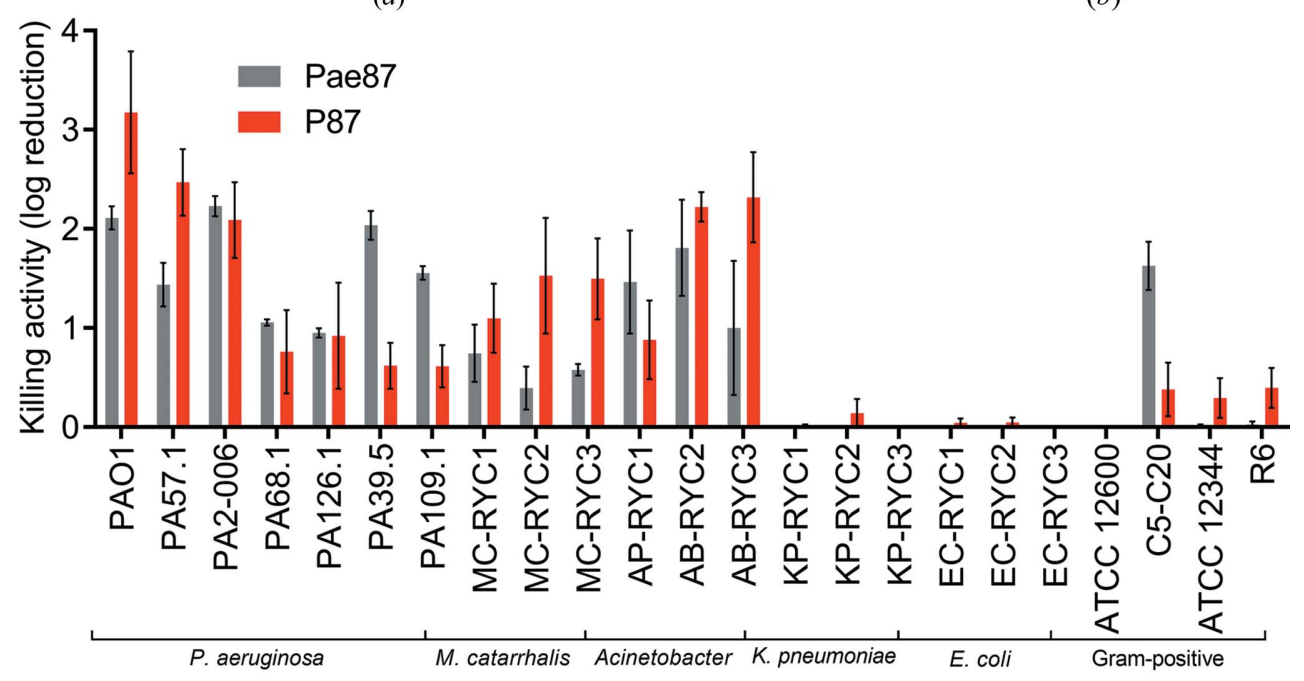

(c)

Figure 8

Antimicrobial activity of P87 peptide. (a) Far-UV circular-dichroism spectra of $20 \mu M$ P87 in $20 \mathrm{~m} M$ NaPiB pH 6.0 with $100 \mathrm{~m} M$ NaCl and different ( $v / v$ ) concentrations of TFE (as indicated in the legend). (b) Turbidity decrease assay of a $10 \mu M$ P87 or Pae87 treatment of PAO1 cell suspensions. (c) Bactericidal activity range of $10 \mu M$ P87. The Pae87 bactericidal range from Vázquez, Blanco-Gañán et al. (2021) is also shown for comparison. 
peptide:bacteria stoichiometry was critical for the killing efficacy of the P87 peptide and, to a lesser extent, of Pae87 (Fig. 11a). In both cases the optimal ratio was achieved at around $10^{7} \mathrm{CFU} \mathrm{ml}{ }^{-1}$ for $10 \mu M$ Pae87 or P87, which roughly corresponds to some $10^{10}$ molecules per cell. The doseresponse curve of Pae87 quickly saturated (Fig. 11b), as has been shown before (Vázquez, Blanco-Gañán et al., 2021). This was speculated to be due to the entrapment of Pae87 molecules within the aggregates. P87 presents a more canonical curve, in line with the effect of the peptide:bacteria ratio, with an increasing activity with higher concentrations (up to a $5 \log$ increase in killing at the maximum concentration tested).

$\mathrm{pH}$ did not seem to be a major determinant of Pae87 activity (Fig. 11c), although the best killing numbers were obtained at mildly acidic $\mathrm{pH}$, as for P87. Ionic strength did not have a remarkable impact at near-physiological concentrations (50$200 \mathrm{mM} \mathrm{NaCl}$ ) for Pae87, but P87 only displayed a relevant killing activity between 50 and $150 \mathrm{mM}$ (Fig. 11d). It is possible that a slight salt concentration might be necessary for proper P87 solubility (in fact, the Aggrescan server detects an N-terminal hotspot for aggregation; de Groot et al., 2012) but that higher concentrations might shield charged residues. This latter hypothesis is supported by the results in Fig. 11(e), in which the activity of $10 \mu M$ P87 was tested in the presence of different concentrations of a non-ionic osmolyte (sorbitol). In these results, the killing activity of the peptide was not affected by increasing concentrations of the non-ionic solute. Finally, PAO1 suspensions that had been treated for $2 \mathrm{~h}$ were subjected to different vortexing treatments before plating to check whether the observed aggregative effect was underestimating the viable cell counts (Fig. 11f). The vortex treatment would disintegrate the aggregates and thus release viable cells that, grouped within the aggregates, might have produced a single colony. The results in Fig. 11(f), however, suggest that this is not the case, i.e. the observed decrease in viable cell counts cannot be solely attributed to mere aggregation, and thus a bactericidal mechanism must be occurring. This is because no significant differences were found in the viable counts after vortexing with respect to the nonvortexed samples, except for the samples treated with Pae87 and vortexed for $15 \mathrm{~min}$. In this latter case, the cell count in fact decreased over the control without vortexing.

\section{Discussion}

Knowledge of the structure and function of phage lysins is a crucial step towards a deeper understanding of how lysins work and their application as engineered antimicrobials. Pae87 has previously been identified and confirmed to be an intrinsically active lysin against a range of Gram-negative pathogens. In this work, this activity has been further inves-

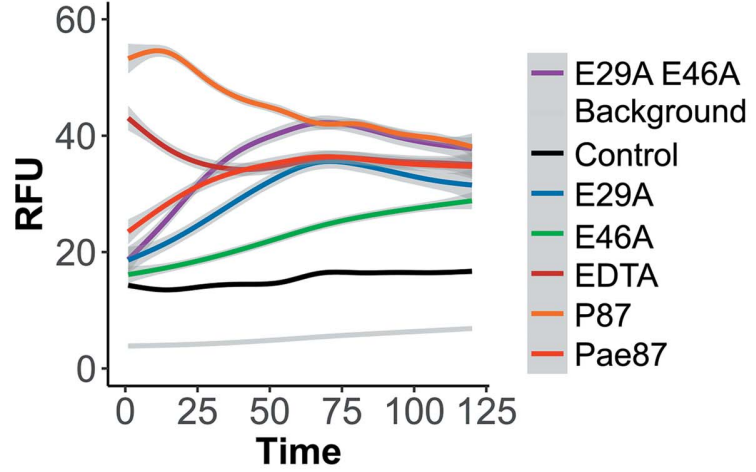

(a)
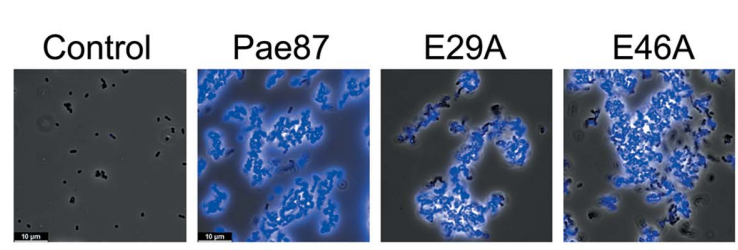

(c)

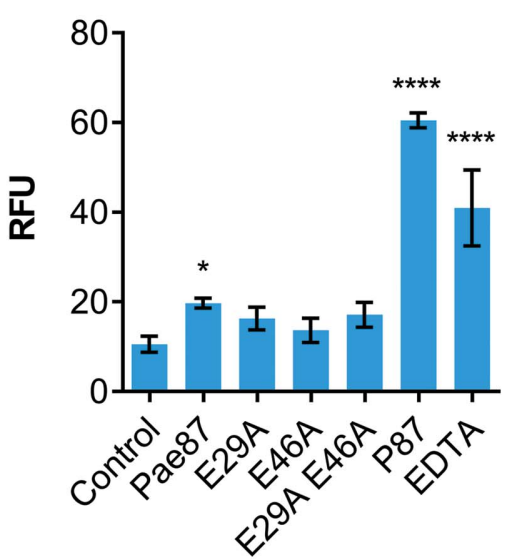

(b)
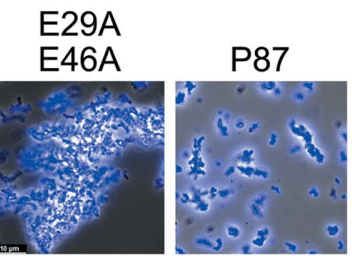

EDTA

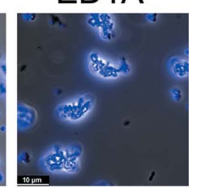

Figure 9

OM permeabilization assays with the fluorescent probe NPN. (a) Generalized additive model estimation of the average tendencies of the NPN fluorescence kinetics (the excitation wavelength was $350 \mathrm{~nm}$ and emission was recorded at $420 \mathrm{~nm}$ ). Estimation was based on three independent replicates; the mean estimation $\pm 95 \%$ confidence interval (shaded grey) is shown for each experimental condition. (b) Comparison of the average NPN signal (minus background fluorescence) after 5 min incubation. The mean \pm standard deviation of three independent replicates is shown; a one-way ANOVA test with a Dunnett post-test was applied to statistically compare each condition with the control (untreated cells in the presence of the probe). *, $p \leq 0.05$; ****, $p \leq 0.0001$. (c) Fluorescence microscopy observation of each experimental condition after $2 \mathrm{~h}$ incubation. Superimpositions of phasecontrast images with blue fluorescence signal observed with an A filter cube (excitation bandpass 340-380 nm) are shown. RFU, fluorescence units relative to the maximum value achieved during the assay. 
tigated in relation to the protein structure to try to unveil the way that Pae87 works as an exogenous antimicrobial.

The structure of Pae87 shows that it is a one-domain protein with the typical $\alpha / \beta$ lysozyme fold. In addition, three $\alpha$-helices are inserted between the $\beta$-strands of the $\beta$-sheet, together forming the $\beta$-lobe. A comparable structure was only found in AP3gp15, a Burkholderia phage lysin. A peptidoglycan fragment was bound to this region of the $\beta$-lobe in the Pae87peptidoglycan crystal structure. Interestingly, the residues predicted to be responsible for the binding of the peptidoglycan fragment were more conserved in representatives from the Muramidase family that lack an additional CWBD than in those that possess one (such as AP3gp15). From these results, the existence of a cell-wall-binding site within the catalytic domain can be proposed. In the absence of a CWBD, this region may be used as a binding site by Pae87 and the other related lysins that contain it, perhaps performing a function in the endogenous lytic activity. In fact, most carbohydrate enzymes known to date possess a CWBD that enables them to approach their substrate, since polysaccharidic substrates are insoluble and thus cannot diffuse into the catalytic centre (Guillén et al., 2010). The observations presented in this work imply that the hypothesis that Gram-negative phage lysins lack or do not need a cell-wall-binding function (Ghose \& Euler, 2020; Vázquez, García et al., 2021) should perhaps be revised once sufficient evidence has been gathered on (i) whether these internal regions within the catalytic domain function as true CWBDs and (ii) how widespread this trait is. To our knowledge, there are few examples in the literature of cell-wall-binding sites contained within the catalytic domain. For example, Bacillus lysin PlyG contains a region located within the NAM-amidase catalytic domain that specifically recognizes B. anthracis spores (Yang et al., 2012). From a functional point of view, this is hardly a comparable case, since the secondary substrate-binding site of PlyG seems to have evolved to recognize a chemically distinct form of the target bacterium that cannot be recognized by the canonical CWBD. A binding region far from the active site has also been
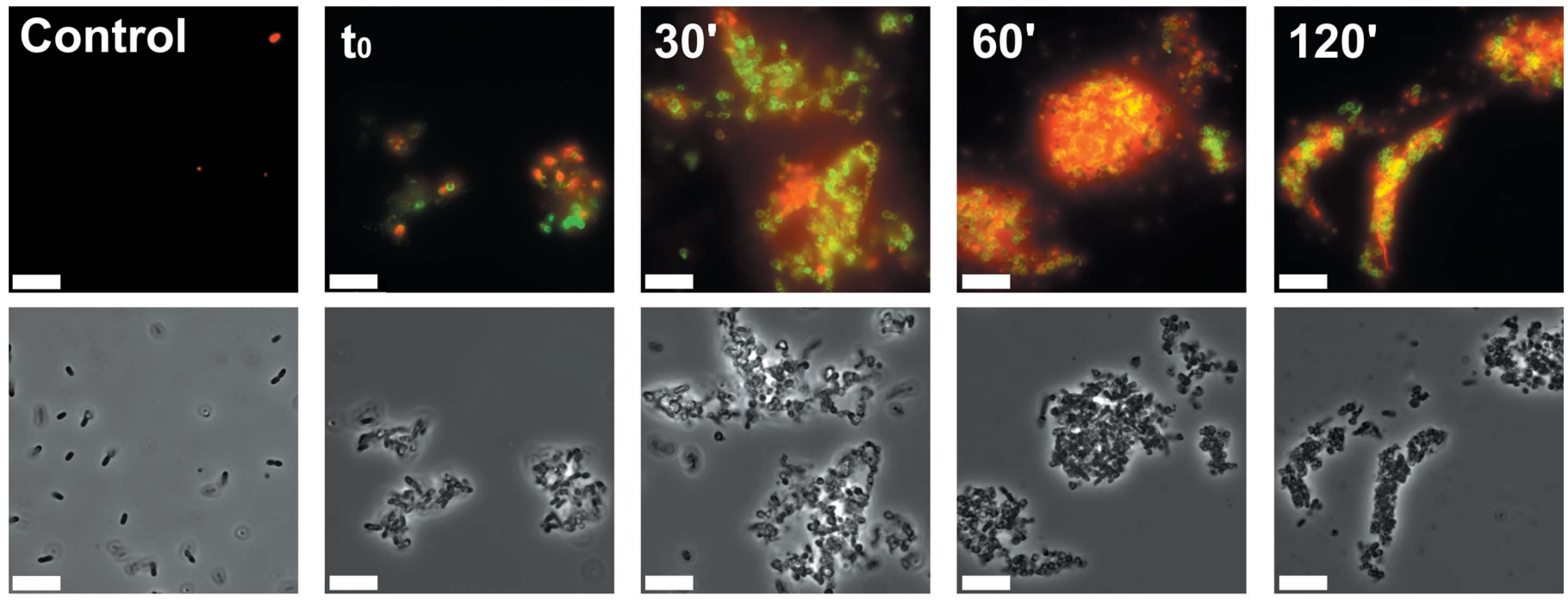

(a)

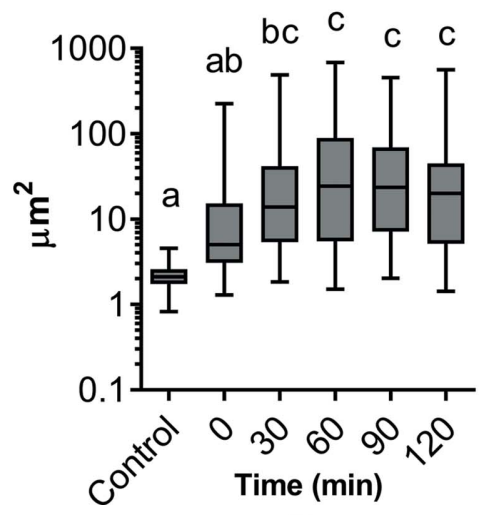

(b)

Figure 10

Microscopic observation of a PAO1 suspension treated with Pae87 over time. (a) Fluorescence and phase-contrast images representative of the observations made during $2 \mathrm{~h}$ of incubation $\left(37^{\circ} \mathrm{C}, 20 \mathrm{~m} M \mathrm{NaPiB} \mathrm{pH} 6.0,150 \mathrm{mM} \mathrm{NaCl}\right)$ of a PAO1 suspension $\left(\sim 10^{8} \mathrm{CFU} \mathrm{ml}{ }^{-1}\right)$ treated with $10 \mu M$ Alexa488-labelled Pae87 and stained with PI. White bars indicate $10 \mu \mathrm{m}$. (b) Time-wise distributions of cells or aggregate areas estimated using the $L A S$ $X$ microscopy image-analysis software for at least ten frames per time point. A one-way ANOVA followed by a Tukey's post-test was applied for multiple comparisons. Distributions marked with different letters are significantly different from each other, while no significant differences were found between those indicated with the same letter. 
proposed at the C-terminal lobe of T4 lysozyme (Grütter \& Matthews, 1982; Kuroki et al., 1993), and in this case it was proposed to bind the peptide moiety of the peptidoglycan, thus providing some specificity to the lytic activity. In contrast, for Pae87 and its close relatives it could be argued that a region with affinity for peptidoglycan has arisen to take over the function of the lacking CWBD, given the differential conservation of the predicted key residues for binding.

Unlike the binding motif, the Pae87 residues of the catalytic cleft are conserved in AP3gp15 (Fig. 2a). Here, the most important residues are Glu29 and Glu46, which are respon- sible for the catalytic activity. Glu29 may correspond to the general acid of other enzymes of the lysozyme family (Wohlkönig et al., 2010). An equivalent to Glu46, the presumed general base, is only found in some lysozymes, such as the peptidoglycan hydrolase Auto (Bublitz et al., 2009) and AP3gp15, although the function of the equivalent Glu46 in the highly homologous AP3gp15 was not studied and in fact it was not pointed out as a possible catalytic residue (Maciejewska et al., 2017). The conserved residues in the MSAs presented in this work have allowed the identification of this glutamic acid and we also showed that an alanine mutant of Glu46 abolishes

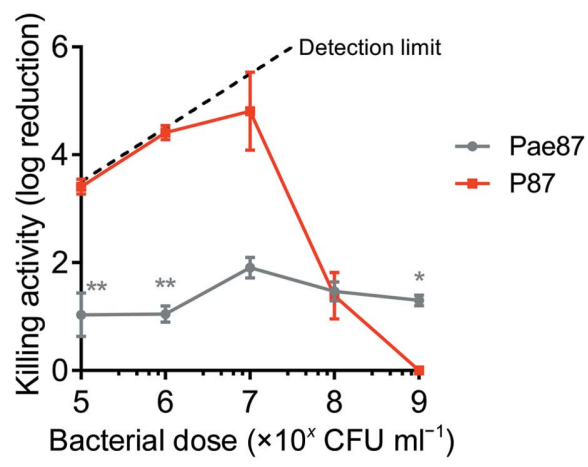

(a)

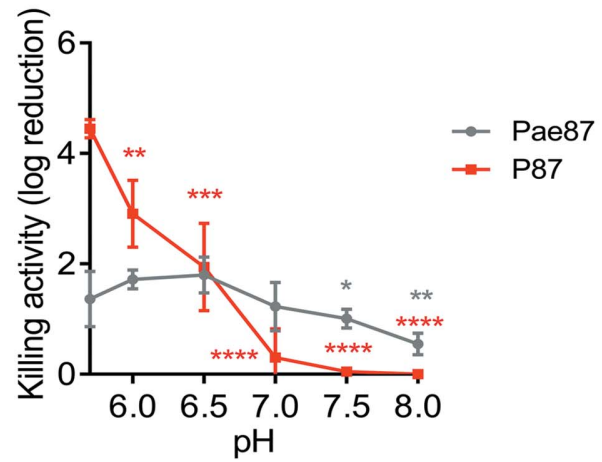

(c)

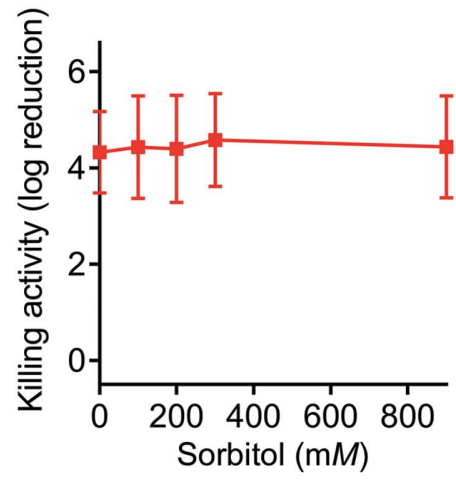

(e)

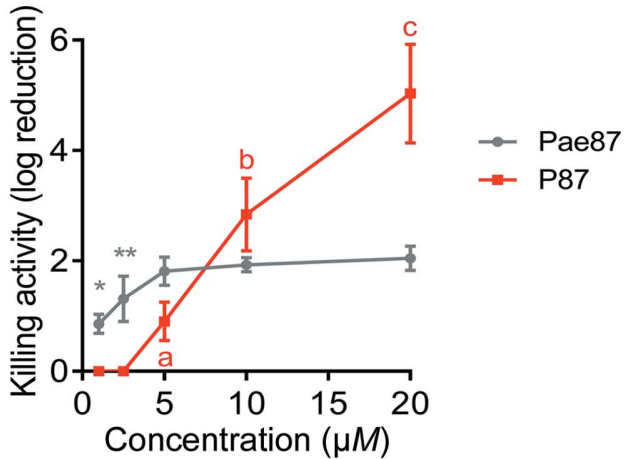

(b)

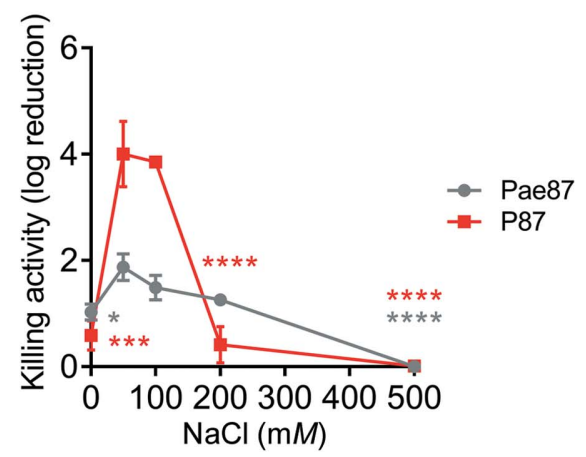

(d)

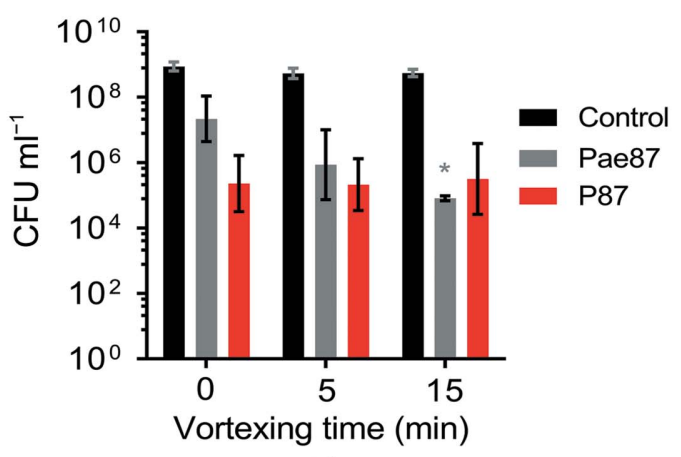

(f)

Figure 11

Parameters of Pae87 and P87 bactericidal activity on P. aeruginosa PAO1. (a) Enzyme:bacteria or peptide:bacteria stoichiometry. (b) Dose-response curves. (c) Variation of killing activity with $\mathrm{pH}$. $(d)$ Variation of killing activity with ionic strength. (e) Variation of killing activity with the concentration of a non-ionic osmolyte (sorbitol), maintaining a fixed concentration of $50 \mathrm{mM} \mathrm{NaCl}$. $(f)$ Viable counts after 0,5 or $15 \mathrm{~min}$ of vortexing. One-way ANOVA with a Dunnett post-test was applied for comparison with the control or the highest value condition: (a) $10^{7} \mathrm{CFU} \mathrm{ml}^{-1}$, (b) $20 \mu M$, (c) $6.5,(d) 50$ or $150 \mathrm{~m} M$, (e) $0 \mathrm{~m} M$ sorbitol and $(f)$ the corresponding viable count without vortex treatment. Unless otherwise stated, the incubation conditions were $20 \mathrm{~m} M \mathrm{NaPiB}$ pH 6.0, $150 \mathrm{~m} M \mathrm{NaCl}, \sim 10^{8} \mathrm{CFU} \mathrm{ml}^{-1}$ PAO1, $10 \mu M$ of the bactericidal compound, $37^{\circ} \mathrm{C}, 2 \mathrm{~h}$. Asterisks indicate $p$-values of significant comparisons $(*, p \leq 0.05 ; * *, p \leq 0.01 ; * * *, p \leq 0.001 ; * * *, p \leq 0.0001)$; nonsignificant comparisons are not indicated. When marked by letters, an allagainst-all multiple comparison was applied with ANOVA plus a Tukey post-test. Different letters indicate significantly different results. 
lysozyme activity in Pae87, therefore confirming the catalytic function of both Glu29 and Glu46. This is further supported by the recent results of Love et al. (2022), which show that a pair of Glu residues are critical for catalysis in the Muramidase family member bimodular LysT84. However, the distance between the Pae 87 glutamates is $16 \AA$, which is longer than the typical distance in inverting glycoside hydrolases (Davies \& Henrissat, 1995). For context, the typical O ..O intercarboxylic distance has been recorded to be $8.5 \pm 2.0 \AA$ on average in inverting $\beta$-glycosidases, while it is shorter ( $4.8 \pm$ 0.3 or $6.4 \pm 0.6 \AA$ ) in $\beta$-glycosidases that use the retaining mechanism (Mhlongo et al., 2014). A putative conclusion from these observations is that the hydrolysis mechanism of Pae87 may operate with net inversion of the anomeric configuration. In fact, Bublitz and coworkers proposed that Auto could move to a closed conformation when it binds to its substrate and thus follow the inverting mechanism of glycoside hydrolysis. The same conformational readjustment and mechanism could occur in Pae87. This hypothesis has been tested for LysT84 by molecular-dynamics simulations, the results of which were compatible with a minimum allowed Glu-Glu distance of around 9-10 $\AA$, which is within the range of inverting glycosidases (Love et al., 2022). While mutating Glu29 and Glu46 dramatically diminished the muralytic efficacy of Pae87, the three mutants (including the E29/E46 double mutant) also retained some residual activity at the maximum concentration tested (less than $50 \%$ of that of the wild-type enzyme), suggesting that other residues may be involved in catalysis (perhaps such conserved residues as His 48 and/or Tyr174), or rather that some residues in the catalytic cleft may take over the function of the mutated amino acid to some degree. Previous analyses of the precise catalytic activity of lysins from the Muramidase family have all concluded that they actually possess a muramidase or lysozyme activity that breaks the glycan chain of the peptidoglycan on the reducing side of MurNAc (Rodríguez-Rubio et al., 2016; Maciejewska et al., 2017). Our results also fit with this proposal. To begin with, correspondence was found between the main degradation peaks detected by RP-HPLC-MS when treating $P$. aeruginosa peptidoglycan with cellosyl, a known lysozyme, and with Pae87. Some of the coincidental main peaks can easily be assigned to peptidoglycan-degradation products. For example, the main peaks with a monoisotopic $\mathrm{m} / \mathrm{z}$ of $\sim 1865$ or $\sim 1845$ are consistent with fragments containing two disaccharides [NAG-(reduced MurNAc)] cross-linked by two tetrapeptides (Ala-Glu-mDAP-Ala). The difference in mass (1865 versus 1845) corresponds to loss of a water molecule, probably from one of the reduced MurNAc residues. The CID results are definitive proof of a muramidase activity, which leaves a MurNAc end susceptible to reduction (conversely, NAG is susceptible to reduction when the glycan strand is degraded by a glucosaminidase activity; Eckert et al., 2006; RodríguezRubio et al., 2016).

On the other hand, the lack of a difference in bactericidal activity between Pae87 and its mutants suggests that the membrane activity is the major determinant of antimicrobial potential in Pae87, rather than the catalytic activity, as pointed out for other intrinsically active lysins (Ibrahim et al., 1996). In this work, a specific AMP-like C-terminal region (P87) with intrinsic membrane-permeabilizing and bactericidal activity has been identified as the part of the enzyme most probably responsible for the aforementioned effect. The crystal structure confirmed that P87 is located on the surface of the protein, supporting the hypothesis that it would be able to directly interact with membranes. Moreover, P87 by itself had an OM permeabilizing activity, as demonstrated by the NPN uptake assay. Nonpolar residues of P87 are mostly buried within Pae87, but not in all cases. For example, Ile164 or even Phe161 are especially exposed (Fig. 7b). The Lys and Arg residues of $\mathrm{P} 87$, on the other hand, are located on the outer surface of the protein and are therefore available for electrostatic interaction with negatively charged elements of the bacterial surface (namely, the phosphate groups of the lipopolysaccharide). Based on the results in this work, including the microscopy images presented in Fig. 10, a mechanism for Pae87 activity from without is proposed: (i) the P87 region of the enzyme would bind to the OM, coating the bacterial surface and then causing the aggregation of adjacent cells, (ii) the membrane-permeabilizing action would then act, perhaps together with the peptidoglycan hydrolysis activity, to disrupt the cell wall and (iii) the leakage of intracellular components and cell death takes place without provoking full disintegration of the bacteria ('lysis'), but rather keeping the cell debris tightly bound in compact aggregates. The decreases in viability after vortexing presented in Fig. 11 are in agreement with the proposal that the aggregates comprise damaged cells (not relatively intact ones) and thus mechanical shaking increased the apparent killing by definitely harming these already damaged bacteria. This 'death without lysis' could be beneficial from the point of view of in vivo therapy, since it would prevent the dissemination of pro-inflammatory factors. Also, bacterial aggregates have previously been shown to be better cleared by the immune system (Ribes et al., 2013; Roig-Molina et al., 2020). However, the true potential of this kind of antimicrobial agent should be tested in vivo to clarify its possible benefits. This proposed mechanism applies to Pae87 when exogenously used as an antibacterial agent. On the other hand, and judging by the previously reported analysis of the phage JG004 lytic cassette (Vázquez, Blanco-Gañán et al., 2021), Pae87 is thought to behave as a canonical endolysin during the infective cycle of the phage, i.e. Pae87 should be exported to the periplasm through holin-formed pores at the late infection stage and thus exert its peptidoglycandegradation activity. Once the peptidoglycan is sufficiently degraded, the putative two-component spanin system should assemble to bring together the inner and outer membrane and create larger pores through which the viral progeny is released, also causing effective lysis of the bacterial host. Within this rather classical framework, the sole function of Pae87 would be to degrade the peptidoglycan. However, this does not exhaustively account for many of the structuralfunctional features that have beeen uncovered in this work. For example, it is still unclear why many Gram-negative lysins have evolved an AMP-like motif (Vázquez, García et al., 
2021). We may speculate that the increased affinity towards the bacterial surface provided by the cationic patch may (i) be beneficial for approaching the insoluble substrate and/or (ii) assist in the permeabilization of the membrane(s) towards effective lysis. In addition, the binding to the cell surface may also be relevant to maintain lysins tightly bound to cell debris, thus preventing a potentially toxic effect in the neighbouring cells. This hypothesis has typically been formulated considering the presence of CWBDs in Gram-positive infecting phage lysins (Loessner et al., 2002), but may also be true in Gram-negative counterparts given that some of them contain motifs that are potentially toxic from without the cells, as demonstrated for Pae87.

Regarding the antimicrobial activity of peptide P87 itself, it was observed to cause an acute lytic effect on $P$. aeruginosa cells. The ability to form amphipathic helices in the presence of TFE, as demonstrated by the circular-dichroism spectra in Fig. 8, would point to the insertion of these helices into the biological membranes and subsequent leakage of the cell contents as the mechanism for P87-mediated killing. The peptide differs in this regard from the poor lytic outcome of Pae87 treatment. This difference may be due to the much smaller size of P87, which could enable it to properly insert into the membranes rather than interact superficially, as it is assumed that Pae87 does.

The maximum detectable killing was observed for $10 \mu M$ P87 at cell concentrations of $10^{7} \mathrm{CFU} \mathrm{ml}{ }^{-1}$ and below, while higher bacterial doses reduced the bactericidal effect, presumably due to a suboptimal number of antimicrobial molecules per cell. This is in agreement with a cooperative mechanism of action where a threshold number of bound peptides is required for bactericidal activity. In addition, the higher activity observed at more acidic $\mathrm{pH}$ values can be explained due to the higher positive charge that both the protein and peptide may have at acidic $\mathrm{pH}$ values, improving the interaction with the negatively charged bacterial surface. Given that an increased activity was observed at $\mathrm{pH} 6.0$ and below, the protonation of histidine residues $\left(\mathrm{p} K_{\mathrm{a}} \simeq 6.0\right)$ is the most plausible explanation. Although P87 was almost inactive at near-physiological $\mathrm{pH}(\sim 7.5)$, the fact that it was highly effective at acidic $\mathrm{pH}$ is relevant for the treatment of infection, since it has been suggested many times that the $\mathrm{pH}$ at the infection site is acidified by a combination of bacterial metabolic activity and immune-system responses (Radovic Moreno et al., 2012; Simmen \& Blaser, 1993). This acidification is especially relevant in certain conditions, such as cystic fibrosis, patients with which are already prone to infections in the respiratory tract, with $P$. aeruginosa being one of the main causative agents in the exacerbation of cystic fibrosis (Poschet et al., 2002). The importance of charge is also manifested by the ionic strength experiments: P87 only displayed a relevant killing activity between 50 and $150 \mathrm{mM} \mathrm{NaCl}$. It is possible that a slight salt concentration might be necessary for proper solubility of P87. Higher concentrations might, however, shield charged residues. This latter hypothesis is supported by the results in the presence of different concentrations of a non-ionic osmolyte (sorbitol), in which the killing activity of the peptide was not affected by an increasing concentration of the solute.

\section{Conclusions}

The three-dimensional structure of Pae87 has been elucidated by X-ray crystallography. This structure provided a basis to propose the presence of a substrate-binding subdomain within the catalytic domain of Pae87. This substrate-binding site is apparently conserved among other enzymes from the same family that lack an independent CWBD and thus may fulfil a compensatory evolutionary function. It was determined that Pae87 is a muramidase, and two acidic residues have been pointed out to be involved in this catalytic activity. However, the antimicrobial activity of Pae87, when exogenously added, was not associated with its catalytic activity, but rather with a nonenzymatic activity on the membranes that most probably resides on a cationic, amphiphilic $\mathrm{C}$-terminal peptide named P87. Such a peptide was proven to be an AMP on its own, and was active against a range of bacteria coincidental to those susceptible to Pae87 surface activity. The activity of P87 was highly dependent on its intrinsic charge and on the peptide: bacteria stoichiometry. Altogether, these results provide further clarity on the structure of a family of Gram negativeactive lysins, revealing evolutionary features with a close relationship to architectural traits of the bacterial hosts. On the other hand, insights have also been provided into the intrinsic antibacterial effect of Pae87 and a novel AMP (P87) has been discovered.

\section{Related literature}

The following reference is cited in the supporting information for this article: Artimo et al. (2012).

\section{Acknowledgements}

The ALBA-CELLS beamline BM13 (XALOC) and Fernando Gil and Xavier Carpena are thanked for providing excellent crystallographic data-collection facilities.

\section{Funding information}

The authors acknowledge the Spanish Ministry of Science and Innovation for grant Nos. SAF2017-88664-R (to PG) and BFU2017-87022-P (to MJvR) funded by MCIN/AEI/10.13039/ 501100011033 and by ERDF A Way of Making Europe, and for the Severo Ochoa program to the CNB-CSIC (SEV 20170712). Additional funding was provided by the Centro de Investigación Biomédica en Red de Enfermedades Respiratorias (CIBERES) to PG, an initiative of the Instituto de Salud Carlos III. RV was the recipient of a predoctoral fellowship from CIBERES.

\section{References}

Abdelkader, K., Gerstmans, H., Saafan, A., Dishisha, T. \& Briers, Y. (2019). Viruses, 11, 96. 
Alvarez, L., Hernandez, S. B., de Pedro, M. A. \& Cava, F. (2016). Methods Mol. Biol. 1440, 11-27.

Artimo, P., Jonnalagedda, M., Arnold, K., Baratin, D., Csardi, G., de Castro, E., Duvaud, S., Flegel, V., Fortier, A., Gasteiger, E., Grosdidier, A., Hernandez, C., Ioannidis, V., Kuznetsov, D., Liechti, R., Moretti, S., Mostaguir, K., Redaschi, N., Rossier, G., Xenarios, I. \& Stockinger, H. (2012). Nucleic Acids Res. 40, W597-W603.

Briers, Y. \& Lavigne, R. (2015). Future Microbiol. 10, 377-390.

Bublitz, M., Polle, L., Holland, C., Heinz, D. W., Nimtz, M. \& Schubert, W. D. (2009). Mol. Microbiol. 71, 1509-1522.

Dams, D. \& Briers, Y. (2019). Adv. Exp. Med. Biol. 1148, 233-253.

Davies, G. \& Henrissat, B. (1995). Structure, 3, 853-859.

Duyvejonck, L., Gerstmans, H., Stock, M., Grimon, D., Lavigne, R. \& Briers, Y. (2021). Antibiotics, 10, 293.

Eckert, C., Lecerf, M., Dubost, L., Arthur, M. \& Mesnage, S. (2006). J. Bacteriol. 188, 8513-8519.

Emsley, P., Lohkamp, B., Scott, W. G. \& Cowtan, K. (2010). Acta Cryst. D66, 486-501.

Evans, P. (2006). Acta Cryst. D62, 72-82.

Evans, P. R. \& Murshudov, G. N. (2013). Acta Cryst. D69, 1204-1214.

Gautier, R., Douguet, D., Antonny, B. \& Drin, G. (2008). Bioinformatics, 24, 2101-2102.

Ghose, C. \& Euler, C. W. (2020). Antibiotics, 9, 74.

Groot, N. S. de, Castillo, V., Graña-Montes, R. \& Ventura, S. (2012). Methods Mol. Biol. 819, 199-220.

Grütter, M. G. \& Matthews, B. W. (1982). J. Mol. Biol. 154, 525-535.

Guillén, D., Sánchez, S. \& Rodríguez-Sanoja, R. (2010). Appl. Microbiol. Biotechnol. 85, 1241-1249.

Helander, I. M. \& Mattila-Sandholm, T. (2000). J. Appl. Microbiol. 88, 213-219.

Holm, L. (2020). Protein Sci. 29, 128-140.

Ibrahim, H. R., Higashiguchi, S., Koketsu, M., Juneja, L. R., Kim, M., Yamamoto, T., Sugimoto, Y. \& Aoki, T. (1996). J. Agric. Food Chem. 44, 3799-3806.

Joosten, R. P., Long, F., Murshudov, G. N. \& Perrakis, A. (2014). IUCrJ, 1, 213-220.

Juanhuix, J., Gil-Ortiz, F., Cuní, G., Colldelram, C., Nicolás, J., Lidón, J., Boter, E., Ruget, C., Ferrer, S. \& Benach, J. (2014). J. Synchrotron Rad. 21, 679-689.

Karplus, P. A. \& Diederichs, K. (2015). Curr. Opin. Struct. Biol. 34, 60-68.

Kezuka, Y., Ohishi, M., Itoh, Y., Watanabe, J., Mitsutomi, M., Watanabe, T. \& Nonaka, T. (2006). J. Mol. Biol. 358, 472-484.

Krissinel, E. (2015). Nucleic Acids Res. 43, W314-W319.

Kuroki, R., Weaver, L. H. \& Matthews, B. W. (1993). Science, 262, 2030-2033.

Lacks, S. \& Hotchkiss, R. D. (1960). Biochim. Biophys. Acta, 39, 508518.

Loessner, M. J., Kramer, K., Ebel, F. \& Scherer, S. (2002). Mol. Microbiol. 44, 335-349.

Loh, B., Grant, C. \& Hancock, R. E. (1984). Antimicrob. Agents Chemother. 26, 546-551.

Love, M. J., Coombes, D., Ismail, S., Billington, C. \& Dobson, R. C. J. (2022). Biochem. J. 479, 207-223.

Low, L. Y., Yang, C., Perego, M., Osterman, A. \& Liddington, R. (2011). J. Biol. Chem. 286, 34391-34403.

Maciejewska, B., Źrubek, K., Espaillat, A., Wiśniewska, M., Rembacz, K. P., Cava, F., Dubin, G. \& Drulis-Kawa, Z. (2017). Sci. Rep. 7, 14501.

Madeira, F., Park, Y. M., Lee, J., Buso, N., Gur, T., Madhusoodanan, N., Basutkar, P., Tivey, A. R. N., Potter, S. C., Finn, R. D. \& Lopez, R. (2019). Nucleic Acids Res. 47, W636-W641.

Mhlongo, N. N., Skelton, A. A., Kruger, G., Soliman, M. E. \& Williams, I. H. (2014). Proteins, 82, 1747-1755.

Murshudov, G. N., Skubák, P., Lebedev, A. A., Pannu, N. S., Steiner, R. A., Nicholls, R. A., Winn, M. D., Long, F. \& Vagin, A. A. (2011). Acta Cryst. D67, 355-367.
Ogata, M., Umemoto, N., Ohnuma, T., Numata, T., Suzuki, A., Usui, T. \& Fukamizo, T. (2013). J. Biol. Chem. 288, 6072-6082.

O'Neill, J. (2016). Tackling Drug-resistant Infections Globally: Final Report and Recommendations. London: The Review on Antimicrobial Resistance. https://amr-review.org/sites/default/files/ 160525_Final\%20paper_with\%20cover.pdf.

Pastagia, M., Schuch, R., Fischetti, V. A. \& Huang, D. B. (2013). J. Med. Microbiol. 62, 1506-1516.

Poschet, J., Perkett, E. \& Deretic, V. (2002). Trends Mol. Med. 8, 512519.

Radovic Moreno, A. F., Lu, T. K., Puscasu, V. A., Yoon, C. J., Langer, R. \& Farokhzad, O. C. (2012). ACS Nano, 6, 4279-4287.

Rau, A., Hogg, T., Marquardt, R. \& Hilgenfeld, R. (2001). J. Biol. Chem. 276, 31994-31999.

Ribes, S., Riegelmann, J., Redlich, S., Maestro, B., de Waal, B., Meijer, E. W., Sanz, J. M. \& Nau, R. (2013). Chemotherapy, 59, 138-142.

Rice, P., Longden, I. \& Bleasby, A. (2000). Trends Genet. 16, 276-277.

Rodríguez-Rubio, L., Gerstmans, H., Thorpe, S., Mesnage, S., Lavigne, R. \& Briers, Y. (2016). Appl. Environ. Microbiol. 82, 4975-4981.

Roig-Molina, E., Sánchez-Angulo, M., Seele, J., García-Asencio, F., Nau, R., Sanz, J. M. \& Maestro, B. (2020). ACS Infect. Dis. 6, 954974.

Sanz-Gaitero, M., Keary, R., Garcia-Doval, C., Coffey, A. \& van Raaij, M. J. (2014). Virol. J. 11, 133.

Shannon, P., Markiel, A., Ozier, O., Baliga, N. S., Wang, J. T., Ramage, D., Amin, N., Schwikowski, B. \& Ideker, T. (2003). Genome Res. 13, 2498-2504.

Simmen, H. P. \& Blaser, J. (1993). Am. J. Surg. 166, 24-27.

Thandar, M., Lood, R., Winer, B. Y., Deutsch, D. R., Euler, C. W. \& Fischetti, V. A. (2016). Antimicrob. Agents Chemother. 60, 26712679.

Vagin, A. \& Teplyakov, A. (2010). Acta Cryst. D66, 22-25.

Vázquez, R., Blanco-Gañán, S., Ruiz, S. \& García, P. (2021). Front. Microbiol. 12, 660403.

Vázquez, R., García, E. \& García, P. (2020). Curated Phage Lysins Collection Including Identifiers, Amino Acid Sequences, Functional Domain Predictions, Architectures and Physicochemical Properties Calculations. http://hdl.handle.net/10261/221469.

Vázquez, R., García, E. \& García, P. (2021). J. Virol. 95, e0032121.

Waterhouse, A. M., Procter, J. B., Martin, D. M., Clamp, M. \& Barton, G. J. (2009). Bioinformatics, 25, 1189-1191.

Wilkins, M. R., Gasteiger, E., Bairoch, A., Sanchez, J. C., Williams, K. L., Appel, R. D. \& Hochstrasser, D. F. (1999). Methods Mol. Biol. 112, 531-552.

Williams, C. J., Headd, J. J., Moriarty, N. W., Prisant, M. G., Videau, L. L., Deis, L. N., Verma, V., Keedy, D. A., Hintze, B. J., Chen, V. B., Jain, S., Lewis, S. M., Arendall, W. B., Snoeyink, J., Adams, P. D., Lovell, S. C., Richardson, J. S. \& Richardson, J. S. (2018). Protein Sci. 27, 293-315.

Winn, M. D., Ballard, C. C., Cowtan, K. D., Dodson, E. J., Emsley, P., Evans, P. R., Keegan, R. M., Krissinel, E. B., Leslie, A. G. W., McCoy, A., McNicholas, S. J., Murshudov, G. N., Pannu, N. S., Potterton, E. A., Powell, H. R., Read, R. J., Vagin, A. \& Wilson, K. S. (2011). Acta Cryst. D67, 235-242.

Winter, G. (2010). J. Appl. Cryst. 43, 186-190.

Wohlkönig, A., Huet, J., Looze, Y. \& Wintjens, R. (2010). PLoS One, 5, e15388.

Yang, H., Wang, D. B., Dong, Q., Zhang, Z., Cui, Z., Deng, J., Yu, J., Zhang, X. E. \& Wei, H. (2012). Antimicrob. Agents Chemother. 56, 5031-5039.

Zallot, R., Oberg, N. \& Gerlt, J. A. (2019). Biochemistry, 58, 41694182.

Zimmermann, L., Stephens, A., Nam, S. Z., Rau, D., Kübler, J., Lozajic, M., Gabler, F., Söding, J., Lupas, A. N. \& Alva, V. (2018). J. Mol. Biol. 430, 2237-2243. 